\title{
Limit theorems for statistics of combinatorial partitions \\ with applications to mean field Bose gas
}

\author{
G. Benfatto, M. Cassandro, I. Merola, and E. Presutti
}

\begin{abstract}
In this paper we study the statistics of combinatorial partitions of the integers, which arise when studying the occupation numbers of loops in the mean field Bose gas. We review the results of Lewis and collaborators $[\mathbf{1 0}][\mathbf{2}]$ and get some more precise estimates on the behavior at the critical point (fluctuations of the condensate component, finite volume corrections to the pressure). We then prove limit shape theorems for the loops occupation numbers. In particular we prove that in a certain range of the parameters, a finite fraction of the total mass is, in the limit, supported by infinitely long loops. We also show that this mass is equal to the mass of the condensed state where all particles have zero momentum.
\end{abstract}

Keywords: Bose condensation, Mean field, Limit theorems, Combinatorial PARTITIONS.

\section{Introduction}

Statistics of combinatorial partitions arises in many areas of science as number theory, combinatorics, probability and statistical mechanics, as illustrated by Vershik in his 1996 paper on the subject, [14].

The problem is about decomposing a positive integer $N \in \mathbb{N}_{+}$into a sum of positive integers, $N=j_{1}+\cdots+j_{k}, k \in \mathbb{N}_{+}$. Let $\left(j_{1}, \ldots, j_{k}\right)$ the sequence of terms in the sum and consider two such sequences equivalent if they differ by a permutation. A partition of $N$ is then the equivalence class of a sequence whose terms sum up to $N$. Following Vershik we describe partitions by 
sequences $\underline{n}=\left\{n_{j}, j \in \mathbb{N}_{+}\right\}$, where $n_{j}$ is the number of elements equal to $j$ in a sequence representative of the partition, thus $\sum_{j} j n_{j}=N$. Statistics enters once we assign a statistical weight to the partitions, the choice of the weights is determined by the particular applications we have in mind and the goal is to derive limit theorems and characterize the typical partitions when $N$ is large.

As in $[\mathbf{1 4}]$ we will consider multiplicative weights, namely we will suppose that the statistical weight of $\underline{n}$ is

$$
w(\underline{n})=\prod_{j=1}^{\infty} w\left(j, n_{j}\right), \quad \underline{n}=\left\{n_{j}, j \in \mathbb{N}_{+}\right\}
$$

where $w(j, \cdot): \mathbb{N}_{+} \rightarrow \mathbb{R}_{+}$.

In the language of statistical mechanics, the assumption restricts the analysis to non interacting systems and we will relax it, to study mean field interactions as well. To make clear the connection with physics, it is convenient to generalize the above context by considering $j$ as an element of some countable space $J$. For instance, a quantum gas of particles in a finite box with Bose-Einstein statistics, can be represented in terms of occupation numbers $\left\{n_{j}, j \in J\right\}$, with $J$ the momentum eigenvalues of a single particle, (which are countably many because particles are in a finite box). In the free case, the equilibrium distribution of such occupation numbers is determined by multiplicative weights of the form (1.1), as it will be discussed in the next section.

Bose condensation is then the phenomenon for which a positive fraction of the total number of particles occupies the state with zero momentum, the fraction converging to a deterministic value in the thermodynamic limit. The other particles are distributed over the remaining momenta and their random distribution, suitably normalized, also converges to a deterministic curve, in the thermodynamic limit. The fraction with zero momentum, 
thought of as a Dirac delta of positive mass added to the remaining distribution, is referred to as the mass of the condensed gas, while the remaining mass is that of the gas in its "normal state". All that happens for suitable values of temperature and density, the theory is very well known and can be found in textbooks and review papers (see for instance $[\mathbf{6}],[\mathbf{1 5}]$ ). The extension to the mean field case is due to Lewis and collaborators (see for instance $[\mathbf{1 0}])$.

Our model is the same free Bose gas discussed so far, but regarded in terms of "loops" which arise when enforcing the symmetry of the wave functions under particles permutations (Bose statistics). To make this paper self contained, in Appendix A, we derive the representation of the canonical partition function for a Bose gas in the loops language. Thus in our scheme $j \in \mathbb{N}_{+}$is the "loop length", representing a cycle with $j$ particles which describe the permutations among particles when imposing the symmetry of the wave function, see Ginibre [5] for a detailed analysis of the model also when inter-particles interactions are present.

Feynman conjectured, [4], that Bose condensation is related to the appearance of long loops, namely a fraction of the total number of particles is concentrated on loops whose length diverges when Bose condensation occurs, and this fraction should be exactly the same as the condensed mass of the gas.

Results of this kind for the free gas, and also in the case when obstacles are present as well, have been proved by Kac and Luttinger [7], [8], Suto $[13]$.

Purpose of our paper is to show that many properties of the free gas are easily and naturally expressed in terms of the loop representation, which in some instances could provide an alternative picture of the system with some advantages over the more usual momentum-occupation representation. We 
will indeed prove very detailed estimates on the statistics of the loops, both in the free and in the mean field case.

In particular our large deviation estimates can be used to extend our analysis in the case the Kac potential with $\gamma^{-1} / L \sim 1$. An extension to a more general class has been obtained in [9].

In Section 2 we present the model. In Section 3 we study the thermodynamics of the mean field Bose gas, showing that the phase diagram can be recovered by solving a variational problem in terms of a free energy functional. We also compute the finite volume corrections to the pressure. In Section 4 we analyze the statistics of the "long loops" whose length goes to

infinity faster than $L^{2}, L$ being the size of the volume. In Section 5 we state large deviation theorems for the "short loops". In Section 6, we prove that the mass density supported by the long loops is equal to the density of the condensed state, where all particles have zero momentum.

Proofs are given in the appendices.

\section{The free and mean field Bose gas}

We will consider the weights $w(j, n)$ in (1.1) as dependent on the parameters $L>0, \beta>0, \lambda \in \mathbb{R}$ and given by the expression

$$
\begin{gathered}
w(j, n)=\frac{1}{n !}\left(\frac{L^{d} a(\beta j, L)}{j} e^{\beta \lambda j}\right)^{n} \\
a(\beta j, L)=\sum_{k \in \mathbb{Z}^{d}} \frac{e^{-(k L)^{2} /(2 \beta j)}}{(2 \pi \beta j)^{d / 2}}
\end{gathered}
$$

The quantity $L^{d} a(\beta j, L)$ is the partition function at temperature $\beta j$ of a free quantum particle of unitary mass in a periodic cubic box of side $L$, 
namely

$$
L^{d} a(\beta j, L)=\sum_{k \in \mathbb{Z}^{d}} e^{-\frac{1}{2} \beta j(2 \pi k / L)^{2}}
$$

The elementary equality (2.3) is proved in Lemma A.2.

By the help of the weights (2.1), we construct three probability measures on $\mathbb{N}_{+}^{\mathbb{N}}$, the canonical, the grand-canonical and the mean field measures. The canonical measure with $N \in \mathbb{N}_{+}$particles is the probability

$$
\mu_{N, L}(\underline{n})=Z_{N, L}^{-1} \mathbf{1}_{\sum j n_{j}=N} w(\underline{n})
$$

the partition function $Z_{N, L}$ being the normalization factor and $w(\underline{n})$ is given by (1.1) with $w\left(j, n_{j}\right)$ as in (2.1) with $\lambda=0$. We are not making explicit the dependence on $\beta$, as it will be kept fixed throughout the sequel. As we will see in Appendix A, $Z_{N, L}$ is equal to the partition function of a Bose gas in a cubic box of length $L$ with periodic boundary conditions and inverse temperature $\beta$.

The grand canonical probability is

$$
P_{\lambda, L}(\underline{n})=\Xi_{\lambda, L}^{-1} w(\underline{n})
$$

where

$$
\Xi_{\lambda, L}=\exp \left\{L^{d} \sum_{j} e^{\beta \lambda j} \frac{a(\beta j, L)}{j}\right\}
$$

and the definition is well posed if $\lambda<0$. Indeed, the r.h.s. of (2.6) diverges for $\lambda \geq 0$ because $a(\beta j, L) \geq 1 / L^{d}$, as follows from (2.3).

Finally, the mean field grand canonical probability is

$$
P_{\lambda, L}^{m f}(\underline{n})=\Xi_{\lambda, L}^{m f}-1 e^{-\beta\left(\sum j n_{j}\right)^{2} /\left(2 L^{d}\right)} w(\underline{n})
$$

(having put equal to 1 the interaction strength). Due to the presence of the interaction, which ensures convergence at infinity, the value of the chemical potential $\lambda$ is now unrestricted. 
To establish the connection of these measures with the Gibbs measures of the Bose gas in the momentum representation, we realize the above processes in the following way. Let $\alpha(\beta j, p, L)>0, j \in \mathbb{N}_{+}, p \in \Pi$, П a countable set, be such that

$$
L^{d} a(\beta j, L)=\sum_{p \in \Pi} \alpha(\beta j, p, L)
$$

We then define new weights

$$
w(j, p, n)=\frac{1}{n !}\left(\frac{\alpha(\beta j, p, L)}{j} e^{\beta \lambda j}\right)^{n}
$$

and call $\mu_{N, L}^{*}(\underline{n}), P_{\lambda, L}^{*}(\underline{n}), P_{\lambda, L}^{m f, *}(\underline{n}), \underline{n}=\left\{n_{j, p}, j \in \mathbb{N}_{+}, p \in \Pi\right\}$, the measures given as in (2.4)-(2.7) with the new weights $w(j, p, n)$ of $(2.9)$ and replacing $\sum_{j} j n_{j} \rightarrow \sum_{j, p} j n_{j, p}$. Calling

$$
n_{j}=\sum_{p \in \Pi} n_{j, p}, \quad n_{p}=\sum_{j>0} j n_{j, p}
$$

a simple combinatorial computation, which is omitted, shows that the laws of the variables $n_{j}$ under $\mu_{N, L}^{*}, P_{\lambda, L}^{*}$ and $P_{\lambda, L}^{m f, *}$ are the same as those under $\mu_{N, L}, P_{\lambda, L}$ and $P_{\lambda, L}^{m f}$.

We will see that some proofs become simpler using the representation (2.10) after a suitable choice of $\alpha(\beta j, p, L)$. To recover the momentum representation, we set $\Pi=\mathbb{Z}^{d}$ and

$$
\alpha(\beta j, p, L)=e^{-\frac{\beta j}{2}\left(\frac{2 \pi p}{L}\right)^{2}}
$$

that satisfies (2.8) (cfr (2.3)). Moreover, the law under $\mu_{N, L}^{*}, P_{\lambda, L}^{*}$ and $P_{\lambda, L}^{m f, *}$ of the variables $n_{p}$ defined in (2.10) is the usual free Bose canonical and grand-canonical and mean field laws of the momentum occupation numbers. Examining for simplicity only the free Bose grand-canonical measure, the 
probability to have $\nu \in \mathbb{N}$ particles of unitary mass with momentum $p$ is

$$
\frac{e^{-\beta \nu\left[-\lambda+\frac{1}{2}\left(\frac{2 \pi p}{L}\right)^{2}\right]}}{\sum_{n \geq 0} e^{-\beta n\left[-\lambda+\frac{1}{2}\left(\frac{2 \pi p}{L}\right)^{2}\right]}}
$$

This is equal to $P_{\lambda, L}^{*}\left(\left\{n_{p}=\nu\right\}\right)$, because,

$$
e^{-\beta \nu\left[-\lambda+\frac{1}{2}\left(\frac{2 \pi p}{L}\right)^{2}\right]}=\sum_{\left\{n_{j, p}, j>0\right\}: \sum_{j} j n_{j, p}=\nu} \prod_{j>0} \frac{1}{n_{j, p} !}\left(\frac{e^{-\beta j\left[-\lambda+\frac{1}{2}\left(\frac{2 \pi p}{L}\right)^{2}\right]}}{j}\right)^{n_{j, p}}
$$

which follows from the combinatorial identity

$$
1=\sum_{\left\{n_{j}, j>0\right\}: \sum_{j} j n_{j}=\nu} \prod_{j>0} \frac{1}{n_{j} !}\left(\frac{1}{j}\right)^{n_{j}}
$$

In turns this identity could be proved by checking that

$$
1=\left.\frac{1}{\nu !} \frac{d^{\nu} f(x)}{d x^{\nu}}\right|_{x=0}, \quad f(x)=\sum_{\left\{n_{j}, j>0\right\}} \prod_{j>0} \frac{1}{n_{j} !}\left(\frac{x^{j}}{j}\right)^{n_{j}}
$$

\section{Thermodynamics of the mean field Bose gas}

By replacing the factorials in $w(\underline{n})$ with the leading terms of the Stirling formula, we obtain the following heuristics for the distribution of the loops occupation numbers at large $L$,

$$
w(\underline{n}) e^{-\beta\left(\sum_{j} j n_{j}\right)^{2} /\left(2 L^{d}\right)} \approx e^{-\beta L^{d} F_{\lambda}(\underline{\rho})}, \quad \underline{\rho}=\left\{j n_{j} / L^{d}, j>0\right\}
$$

where $F_{\lambda}(\underline{\rho}):[0,+\infty)^{\mathbb{N}_{+}} \rightarrow \mathbb{R} \sqcup\{+\infty\}$, is defined by the expression

$$
F_{\lambda}(\underline{\rho})=\frac{1}{2}\left(\sum_{j} \rho_{j}\right)^{2}-\lambda \sum_{j} \rho_{j}-\frac{S(\underline{\rho})}{\beta}
$$




$$
S(\underline{\rho})=-\sum_{j=1}^{\infty} \frac{\rho_{j}}{j}\left(\log \frac{\rho_{j}}{\rho_{j}^{*}}-1\right), \quad \rho_{j}^{*}=\frac{1}{(2 \pi \beta)^{d / 2} j^{d / 2}}
$$

Besides the above heuristic derivation, the functional $F_{\lambda}(\underline{\rho})$ has an important role in the sequel. Indeed, as suggested by (3.1) and proved in this paper, in the thermodynamic limit $L \rightarrow \infty$, the distribution concentrates on the minimizers of $F_{\lambda}$, thus reducing the computation of the "macroscopic observables" to variational problems for the "limit functional" $F_{\lambda}$. In particular this applies to the thermodynamic potentials. Indeed, interpreting $F_{\lambda}(\underline{\rho})$ as the Gibbs thermodynamic potential and applying the corresponding version of the second principle of thermodynamics, we have the following expression for the equilibrium thermodynamical pressure

$$
\pi(\lambda):=-\inf _{\underline{\rho}} F_{\lambda}(\underline{\rho})
$$

The validity of such an interpretation is confirmed by equality with the mean field grand canonical pressure:

$$
\pi(\lambda)=\lim _{L \rightarrow \infty} \frac{1}{\beta L^{d}} \ln \Xi_{\lambda, L}^{m f}
$$

According to thermodynamics, the free energy functional which corresponds to the Gibbs potential $F_{\lambda}(\underline{\rho})$ is $F_{\lambda}(\underline{\rho})+\lambda\left\{\sum \rho_{j}\right\}$; we can then use the latter to define the equilibrium thermodynamical free energy:

$$
a(u):=\inf _{\underline{\rho}: \sum_{j} \rho_{j}=u}\left\{F_{\lambda}(\underline{\rho})+\lambda u\right\}
$$

The validity of (3.6) follows from equality with the mean field canonical free energy, which can be written, if $Z_{N_{L}, L}^{m f}$ denotes the mean field canonical partition function:

$$
a(u)=-\lim _{\substack{L \rightarrow \infty \\ N_{L} / L^{d} \rightarrow u}} \frac{1}{\beta L^{d}} \ln Z_{N_{L}, L}^{m f}
$$


and thermodynamic consistency follows from checking that $a(u)$ is the Legendre transform of $\pi(\lambda)$.

(3.4)-(3.7) show that the thermodynamics of the mean field Bose gas is the same thermodynamics of the free energy functional $F_{\lambda}$, which can be quite explicitly computed. All that, including the proofs of (3.4)-(3.7), are reported in appendix $\mathrm{G}$.

The thermodynamics of the Bose gas (in the free and in the mean field cases) is very well known and does not need to be discussed again here, but its features in terms of loops are not so familiar and, on the other hand, quite interesting and transparent. Recall first that in the free gas there is, in any dimension $d \geq 3$, a Bose condensation characterized by the existence of a critical density $u^{*}$, so that the free energy

$$
a^{0}(u):=a(u)-\frac{u^{2}}{2}
$$

(i.e. the mean field free energy $a(u)$ minus the mean field energy when the particles density is $u$ ) is constant past $u^{*}$ :

$$
a^{0}(u)=a^{0}\left(u^{*}\right)
$$

Such a property is indeed verified by $a^{0}(u)$ as defined by (3.8) with $a(u)$ as in (3.6), which means, recalling (2.4) and (3.7), that, if $u \geq u^{*}$ and [.] denotes the integer part,

$$
\lim _{L \rightarrow \infty} \lim _{\substack{N_{L}=\left[L^{d} u\right] \\ N_{L}^{*}=\left[L^{d} u^{*}\right]}} \frac{1}{\beta L^{d}} \ln \left\{\frac{Z_{N_{L}, L}}{Z_{N_{L}^{*}, L}}\right\}=0
$$

(3.10) shows that the ratio $Z_{N_{L}, L} / Z_{N_{L}^{*}, L} \rightarrow 1$ (in a very weak sense, indeed). The closeness to equality before the limit is an indication of validity of the Bose condensation phenomenon in finite volumes. We have a result (proved in appendix B) which shows that the infinite volume description is very accurate: 
Theorem 3.1. Let $d \geq 3$ and

$$
u^{*}=\sum_{j>0} \rho_{j}^{*}
$$

Then, given any density $u>u^{*}$ and any two sequences, $N_{L}$ and $N_{L}^{*}$, such that $N_{L}=\left[L^{d} u\right]$ and $N_{L}^{*}=\left[L^{d} u^{*}\right]$, there is a constant $c_{0}$, only dependent on $d$, such that

$$
\lim _{L \rightarrow \infty} \frac{Z_{N_{L}, L}}{Z_{N_{L}^{*}, L}}=c_{0}
$$

Moreover, there exists another dimension dependent constant $c_{1}$, such that, if $\lambda>u^{*}$,

$$
\lim _{L \rightarrow \infty} \frac{\Xi_{\lambda, L}^{m f} e^{-\frac{\beta \lambda^{2} L^{d}}{2}}}{\Xi_{u^{*}, L}^{m f} e^{-\frac{\beta\left(u^{*}\right)^{2} L^{d}}{2}}}=c_{1}
$$

Let us now describe the condensation phenomenon in terms of loops, starting from the analysis of the functional $F_{\lambda}(\underline{\rho})$. In any dimension $d \geq 3$, there is a critical chemical potential

$$
\lambda^{*}=\sum_{j>0} \rho_{j}^{*}=u^{*}
$$

and, for $\lambda>\lambda^{*}$, the inf in (3.4) is not a minimum, but (cfr. appendix G) it is obtained by any minimizing sequence $\underline{\rho}^{(n)}=\left\{\rho_{j}^{(n)}, j>0\right\}$, such that, for any fixed $j$,

$$
\lim _{n \rightarrow \infty} \rho_{j}^{(n)}=\rho_{j}^{*}
$$

while:

$$
\rho(\lambda):=\lim _{n \rightarrow \infty} \sum_{j>0} \rho_{j}^{(n)}=\sum_{j>0} \rho_{j}^{*}+\left(\lambda-\lambda^{*}\right)=\lambda
$$

(3.15)- (3.16) show that a fraction $\lambda-\lambda^{*}$ of the total mass $\rho(\lambda)$ concentrates on "infinite loops". The phenomenon is absent for $\lambda \leq \lambda^{*}$, where instead 
the r.h.s. of (3.4) has a unique minimizer $\rho(\lambda)=\left\{\rho_{j}(\lambda), j>0\right\}$, where

$$
\rho_{j}(\lambda)=\rho_{j}^{*} e^{\beta j \lambda_{0}(\lambda)}
$$

and $\lambda_{0}(\lambda)$ is strictly positive for $\lambda<\lambda^{*}$, and $=0$ otherwise. Thus the total mass of the fluid is

$$
\rho(\lambda)= \begin{cases}\sum_{j>0} \rho_{j}(\lambda), & \text { if } \lambda \leq \lambda^{*} \\ \sum_{j>0} \rho_{j}^{*}+\left(\lambda-\lambda^{*}\right), & \text { if } \lambda \geq \lambda^{*}\end{cases}
$$

and no mass concentrates on infinite loops for $\lambda \leq \lambda^{*}$. Note that, by (3.14), $\rho\left(\lambda^{*}\right)=\lambda^{*}$.

The validity of the above interpretation follows from following theorem, which is a corollary of the large deviation estimates proved in Appendix E.

Theorem 3.2. For any $\lambda,(3.19)$, (3.20) and (3.21) below hold.

$$
\begin{gathered}
\lim _{L \rightarrow \infty} P_{\lambda, L}^{m f}\left(\left|\frac{j n_{j}}{L^{d}}-\rho_{j}(\lambda)\right|>\delta\right)=0, \quad \forall \delta>0 \\
\lim _{L \rightarrow \infty} P_{\lambda, L}^{m f}\left(\left|\sum_{j \leq J(L)}\left\{\frac{j n_{j}}{L^{d}}-\rho_{j}(\lambda)\right\}\right|>\delta\right)=0, \quad, \quad \forall \delta>0
\end{gathered}
$$

independently of the choice of $J(L)$, provided $J(L)$ is an increasing function of $L$ and $J(L) \leq L^{2}$.

$$
\lim _{L \rightarrow \infty} P_{\lambda, L}^{m f}\left(\left|\sum_{j \geq 1}\left\{\frac{j n_{j}}{L^{d}}-\rho(\lambda)\right\}\right|>\delta\right)=0 \quad, \quad \forall \delta>0
$$

While the statements relative to global quantities, like pressure, free energy and total number of particles are known in the literature, the results on the way the mass distributes among the different loops are new for the 
mean field interaction; Suto, $[\mathbf{1 3}]$, has analogous results in the context of the canonical free measure.

But all this is not really in the focus of our study, which is rather aimed at relaxing the assumption of mean field, for instance considering Kac potentials, with the hope that the loops language may provide some simplification. In this perspective it is important to derive sharp estimates on the deviations of the densities in (3.19), (3.20) and (3.21), which have been used in $[\mathbf{9}]$ to prove the occurrence of Bose condensation with Kac potentials in suitable scaling limits and to get non trivial estimates for the low momenta distribution in the condensed region for a class of long but finite range potential. Results and proofs can be found in Section 5 and Appendix B.

The rate functions of the large deviations of the above macroscopic quantities are faithfully described by the functional $F_{\lambda}(\underline{\rho})$, whose suitably constrained minima give the correct large deviations rate functions. Thus, like in the case of the thermodynamical potentials, the analysis of the functional $F_{\lambda}(\rho)$ gives the right answer.

The functional $F_{\lambda}(\underline{\rho})$ is instead inadequate for studying how the mass of the condensed fluid (in the Bose condensation regime $\lambda>\lambda^{*}$ ) distributes among the long loops. The issue is discussed in the next section.

\section{Distribution of long loops}

To study the Bose condensation phenomenon, we restrict to $d \geq 3$ and to $\lambda>\lambda^{*}$. Then, see (3.18)-(3.21) and (3.14), the total mass (after the thermodynamic limit) is $\rho(\lambda)=u^{*}+\left(\lambda-\lambda^{*}\right), u^{*}$ is the mass of the "normal 
fluid" and $\left(\lambda-\lambda^{*}\right)$ of the condensed one. By (3.20),

$$
\lim _{L \rightarrow \infty} P_{\lambda, L}^{m f}\left(\left|\sum_{j \leq L^{2}} \frac{j n_{j}}{L^{d}}-u^{*}\right|>\delta\right)=0 \quad, \quad \forall \delta>0
$$

which shows that in finite volumes the mass of the normal fluid is essentially carried by loops with length $\leq L^{2}$, while the mass of the condensed concentrates on loops of length $>L^{2}$ :

$$
\lim _{L \rightarrow \infty} P_{\lambda, L}^{m f}\left(\left|\sum_{j>L^{2}} \frac{j n_{j}}{L^{d}}-\left(\lambda-\lambda^{*}\right)\right|>\delta\right)=0 \quad, \quad \forall \delta>0
$$

Actually most of the mass is on loops whose length is a fraction of the whole volume:

$$
\lim _{L \rightarrow \infty} E_{\lambda, L}^{m f}\left(\frac{1}{L^{d}} \sum_{j>L^{2}}^{\delta L^{d}} j n_{j}\right)=\delta
$$

Furthermore the number $\tilde{X}_{L}$ of loops larger than $L^{2}$ goes like $\ln L$ and becomes deterministic in the limit $L \rightarrow \infty$ (i.e. $\tilde{X}_{L} / \ln L \rightarrow a>0$ ), while the cardinality of the subset of loops larger than $\delta L^{d}, \delta>0$, is finite and has a non trivial (i.e. non deterministic) limit distribution.

We summarize this result in the following Theorem proved in Appendix F, where we use the following notation:

$$
\begin{gathered}
y_{\delta, L} \equiv \frac{1}{L^{d}} \sum_{j \geq \delta L^{d}} j n_{j} \quad, \quad X_{\delta, L} \equiv \sum_{j \geq \delta L^{d}} n_{j} \quad, \quad X_{L} \equiv \frac{1}{\log L} \sum_{j \geq L^{2}} n_{j} \\
j_{\max }:=\max \left\{j: n_{j}>0\right\}
\end{gathered}
$$

Theorem 4.1. Suppose that $\lambda>\lambda^{*}$ and $0<\delta<\lambda-\lambda^{*}$, then

$$
\begin{gathered}
\lim _{L \rightarrow \infty} E_{\lambda, L}^{m f}\left(y_{\delta, L}\right)=\lambda-\lambda^{*}-\delta \\
\lim _{L \rightarrow \infty}\left\{E_{\lambda, L}^{m f}\left(y_{\delta, L}^{2}\right)-E_{\lambda, L}^{m f}\left(y_{\delta, L}\right)^{2}\right\}=\frac{1}{2} \delta^{2}
\end{gathered}
$$




$$
\begin{gathered}
\lim _{L \rightarrow \infty} E_{\lambda, L}^{m f}\left(X_{\delta, L}\right)=\log \frac{\lambda-\lambda^{*}}{\delta}, \quad \lim _{L \rightarrow \infty} E_{\lambda, L}^{m f}\left(X_{L}\right)=d-2 \\
\lim _{L \rightarrow \infty}\left\{\sqrt{\log L}\left[E_{\lambda, L}^{m f}\left(X_{L}^{2}\right)-E_{\lambda, L}^{m f}\left(X_{L}\right)^{2}\right]\right\}=d-2 \\
\lim _{L \rightarrow \infty}\left\{E_{\lambda, L}^{m f}\left(X_{\delta, L}^{2}\right)-E_{\lambda, L}^{m f}\left(X_{\delta, L}\right)^{2}\right\}= \\
=D_{\delta}=\log \frac{\lambda-\lambda^{*}}{\lambda-\lambda^{*}-\delta}\left(1-\log \frac{\lambda-\lambda^{*}}{\delta}\right)+ \\
+\int_{\delta}^{\lambda-\lambda^{*}-\delta} \frac{d x}{x}\left(1-\log \frac{\lambda-\lambda^{*}}{\lambda-\lambda^{*}-x}\right) \\
\frac{D_{\delta}}{\log \left[\left(\lambda-\lambda^{*}\right) / \delta\right]} \frac{1}{\delta \rightarrow 0} 1
\end{gathered}
$$

Furthermore, for any $\xi \in(1 / 2,1]$,

$$
\lim _{L \rightarrow \infty} P_{\lambda, L}^{m f}\left(\frac{j_{\max }}{L^{d}}>\xi\left(\lambda-\lambda^{*}\right)\right)=-\ln \xi
$$

Suto, [13], has already a proof of (4.3) in the free canonical case, but he has not analyzed in detail the statistics of long loops.

\section{Small and large deviations}

\subsection{Small deviations.}

Theorem 5.1 (small fluctuations for $N$ ). Let

$$
\sigma^{2}:=\lim _{L \rightarrow \infty} \frac{1}{L^{d}} E_{\lambda, L}^{m f}\left(\left[\sum_{j>0} j\left(n_{j}-\left\langle n_{j}\right\rangle\right)\right]^{2}\right), \quad\left\langle n_{j}\right\rangle:=E_{\lambda, L}^{m f}\left(n_{j}\right)
$$

Then, if $\lambda_{0}(\lambda)$ is defined as in Appendix $G$,

$$
\sigma^{2}=\left[\beta+\frac{\mathbf{1}_{\left\{\lambda<\lambda^{*}\right\}}}{\sum_{j} \rho_{j}^{*} j e^{\lambda_{0}(\lambda) \beta j}}\right]^{-1}
$$


for any $\lambda$, if $d=3,4$, and for $\lambda \neq \lambda^{*}$, if $d \geq 5$. Moreover, under the same conditions on $\lambda$, the function

$$
\Im(v):=\inf _{\underline{\rho}: \sum \rho_{j}=\rho(\lambda)+v} F_{\lambda}(\underline{\rho})-\inf _{\underline{\rho}} F_{\lambda}(\underline{\rho})
$$

with $\rho(\lambda)$ given by (3.18), is twice differentiable in $v=0$ and

$$
\sigma^{2}=\left(\beta\left[\frac{d^{2} \Im(v)}{d v^{2}}\right]_{v=0}\right)^{-1}
$$

Proof. The value of $\sigma^{2}$ is calculated in Appendix C, Theorem C.2, in the case $\lambda \geq \lambda^{*}$; the case $\lambda<\lambda^{*}$ could be treated in a similar (simpler) way. The relation with the free energy functional, equation (5.4), follows from (I.6) of Appendix I.

Remark: If $d=3$ and $\lambda \neq \lambda^{*}$, equation (5.2) was already obtained by [2], but the case $\lambda=\lambda^{*}$ seems new. To check that the expression given in $[2]$ coincides with (5.2) for $\lambda \neq \lambda^{*}$, it is sufficient to note that, since $\rho(\lambda)=\lambda-\lambda_{0}(\lambda)$ and, in $d=3, \rho_{j}^{*}=\frac{1}{(2 \pi \beta j)^{3 / 2}}$

$$
\begin{aligned}
\sum_{j} \rho_{j}^{*} \beta j e^{\lambda_{0}(\lambda) \beta j} & =\frac{\beta}{(2 \pi \beta)^{3 / 2}} \sum_{j} \frac{1}{j^{1 / 2}} e^{(\lambda-\rho(\lambda)) \beta j} \\
& \equiv \frac{\beta}{(2 \pi \beta)^{3 / 2}} \mathfrak{g}_{1 / 2}(\lambda-\rho(\lambda))
\end{aligned}
$$

where $\mathfrak{g}_{1 / 2}(\mu)$ is defined in formulas $17-18$ of $[\mathbf{2}]$.

We will also study deviations of other macroscopic quantities. In particular we will consider the following sets:

$$
V_{1}:=\mathbb{Z}^{+} \quad V_{2}:=\{\ell\} \quad V_{3}:=\{J(L)\} \quad V_{4}:=\{1,2, \ldots, J(L)\}
$$

with $J(L)$ as in Theorem 3.2, namely $J(L) \in \mathbb{N}^{+}$is an increasing function of $L$, such that $\lim _{L \rightarrow \infty} J(L)=\infty$ and $\lim _{L \rightarrow \infty} J(L) / L^{2}<\infty$. 
For $k=1, . ., 4$, we then define

$$
\begin{aligned}
& \mathcal{A}_{L, \delta}^{(k)}(v) \equiv \mathcal{A}_{L, \delta}^{(k)}:=\left\{\underline{n}: \frac{1}{L^{d}} N^{(k)} \in\left(\sum_{j \in V_{k}} \rho_{j}(\lambda)+v-\delta, \sum_{j \in V_{k}} \rho_{j}(\lambda)+v+\delta\right)\right\} \\
& A_{L}^{(k)}(v) \equiv A_{L}^{(k)}:=\left\{\underline{\rho}: \sum_{j \in V_{k}} \rho_{j}=\sum_{j \in V_{k}} \rho_{j}(\lambda)+v\right\}
\end{aligned}
$$

with

$$
N^{(k)}=\sum_{j \in V_{k}} j n_{j}
$$

and $\rho_{j}(\lambda)$ as in (3.17).

The small deviations for $N^{(k)}, k \neq 1, N^{(k)}$ as in (5.9), are discussed in Appendix D for $\lambda>\lambda^{*}$. The relation of the corresponding covariances with the free energy functional goes along the same lines of Appendix I and we omit it.

\subsection{Large deviations.}

In this subsection we will express the rate functions of large deviations for the quantities (5.7), (5.8) in terms of variational problems for the limit functional with corresponding constraints.

Theorem 5.2. For any $\lambda$, if $k=1$, and for any $\lambda \neq \lambda^{*}$, if $k>1$,

$$
\lim _{\delta \rightarrow 0} \lim _{L \rightarrow \infty}\left[\frac{\frac{1}{\beta L^{d}} \ln P_{\lambda, L}^{m f}\left(\mathcal{A}_{L, \delta}^{(k)}\right)}{-\inf _{\underline{\rho} \in A_{L}^{(k)}} F_{\lambda}(\underline{\rho})+\inf _{\underline{\rho}} F_{\lambda}(\underline{\rho})}\right]=1
$$

Proof. The proof of this Theorem in the case $k=4$ and $\lambda>\lambda^{*}$ (the most interesting case) follows from Theorem E.3 in Appendix E and Appendix $H$. The other cases can be treated along the same lines. 
Remark: The case $\lambda=\lambda^{*}$ is more involved, if $k>1$, so we did not study it in detail, but we think that Theorem 5.2 is still valid.

Corollary 5.3 (large deviations for $\left.N^{(1)} \equiv N\right)$.

$$
\lim _{\delta \rightarrow 0} \lim _{L \rightarrow \infty} \frac{\ln P\left(\mathcal{A}_{L, \delta}^{(1)}\right)}{\beta L^{d}}=-\bar{t}(\rho(\lambda)+v)+\frac{v^{2}}{2}+\rho(\lambda) v+\pi_{\lambda_{0}(\lambda+\bar{t})}^{0}-\pi_{\lambda_{0}(\lambda)}^{0}
$$

where $\lambda_{0}(\lambda)$ is defined in (G.5), $\pi_{\lambda_{0}}^{0}$ is the pressure of the free system with chemical potential $\lambda_{0}$ (cfr. equation (G.2)) and $\bar{t}$ is the solution of the equation $\bar{t}=\lambda_{0}(\lambda+\bar{t})-\lambda_{0}(\lambda)+v$.

Remark: If $\lambda>\lambda^{*}$ and $\lambda+v>\lambda^{*}$, then $\rho(\lambda)=\lambda, \lambda_{0}=0$ and the expression on the r.h.s. of (5.11) becomes $-\frac{v^{2}}{2}$.

Corollary 5.4 (large fluctuations for $N^{(2)}$ and $\left.N^{(3)}\right)$. If $\lambda \geq \lambda^{*},-\rho_{\ell}^{*}<$ $v<\lambda-\lambda^{*}$ and we define $\theta:=\frac{v}{\rho_{\ell}^{*}}$, then

$$
\lim _{\delta \rightarrow 0} \lim _{L \rightarrow \infty} \frac{1}{\beta L^{d}} \ln P\left(\mathcal{A}_{L, \delta}^{(2)}\right)=-\frac{1}{\beta} \frac{v}{\ell}\left[\left(\theta^{-1}+1\right) \ln (1+\theta)-1\right]
$$

while, if $\lambda \geq \lambda^{*}$ and $v>\lambda-\lambda^{*}$,

$$
\begin{aligned}
& \lim _{\delta \rightarrow 0} \lim _{L \rightarrow \infty} \frac{1}{\beta L^{d}} \ln P\left(\mathcal{A}_{L, \delta}^{(2)}\right) \\
& =-\frac{1}{\beta} \frac{v}{\ell}\left[\left(\theta^{-1}+1\right) \ln (1+\theta)-1\right]+\frac{\lambda_{0}^{2}}{2}-\lambda_{0}+\pi_{\lambda_{0}}^{0}-\pi_{0}^{0}
\end{aligned}
$$

where $\lambda_{0}=\lambda_{0}\left(\lambda-\rho_{\ell}^{*}-v\right)$.

If $\lambda>\lambda^{*}$ and $v>0$ :

$$
\lim _{\delta \rightarrow 0} \lim _{L \rightarrow \infty} \frac{J(L)}{\beta L^{d} \ln J(L)} \ln P\left(\mathcal{A}_{L, \delta}^{(3)}\right)=-\frac{v d}{2}
$$

For $\lambda>\lambda^{*}$ and $v<0$ we get:

$$
\lim _{\delta \rightarrow 0} \lim _{L \rightarrow \infty} \frac{1}{\beta L^{d}} \ln P\left(\mathcal{A}_{L, \delta}^{(3)}\right)=-\frac{c(v) d}{2}
$$

where $c(v)>0$ and vanishes as $v \rightarrow 0$ (cfr. corollary 5.5) 
Proof. By Theorem 5.2 and Appendix H

$$
\lim _{\delta \rightarrow 0} \lim _{L \rightarrow \infty} \frac{1}{\beta L^{d}} \ln P\left(\mathcal{A}_{L, \delta}^{(2)}\right)=-\bar{t}\left(\rho_{\ell}(\lambda)+v\right)+\pi_{\lambda, \bar{t}}-\pi_{\lambda}
$$

where $\bar{t}$ is the solution of the equation $\rho_{\ell}(\lambda) e^{\bar{t} \beta \ell}=\rho_{\ell}(\lambda)+v$. The solution does exist when $v>-\rho_{\ell}(\lambda)$ and is given by:

$$
\bar{t}=\frac{1}{\beta \ell} \ln \left(\frac{\rho_{\ell}(\lambda)+v}{\rho_{\ell}(\lambda)}\right)
$$

When $\lambda>\lambda_{*}$ and $-\rho_{\ell}^{*}<v<\lambda-\lambda^{*}$, we see that $\tilde{\pi}_{\lambda, \bar{t}}-\pi_{\lambda}=\frac{\lambda^{2}}{2}+$ $\frac{1}{\beta} \sum_{j} \frac{\left(\rho_{j}^{*}+v \mathbf{1}_{j=\ell}\right)}{j}-\frac{\lambda^{2}}{2}-\frac{1}{\beta} \sum_{j} \frac{\rho_{j}^{*}}{j}=\frac{v}{\beta \ell}$, so that, defining $\theta:=\frac{v}{\rho_{\ell}^{*}}$,

$$
\lim _{\delta \rightarrow 0} \lim _{L \rightarrow \infty} \frac{1}{\beta L^{d}} \ln P\left(\mathcal{A}_{L, \delta}^{(2)}\right)=-\frac{1}{\beta} \frac{v}{\ell}\left[\left(\theta^{-1}+1\right) \ln (1+\theta)-1\right]
$$

while, if $v>\lambda-\lambda^{*}$, we get an extra term coming from the difference $\tilde{\pi}_{\lambda, \bar{t}}-\pi_{\lambda}$.

(5.14) is a direct consequence of (5.12), obtained in the limit $\theta \rightarrow \infty$.

Notice that, when $\lambda>\lambda^{*}$, in the limit $L \rightarrow \infty$, the fluctuation of $\rho_{\ell}$ has the law of a free Poisson distribution with parameter $\rho_{\ell}^{*}$.

Corollary 5.5 (large deviations for $N^{(4)}$ ). For $\lambda>\lambda^{*} ; v>0$ :

$$
\lim _{\delta \rightarrow 0} \lim _{L \rightarrow \infty} \frac{J(L)}{\beta L^{d} \ln J(L)} \ln P\left(\mathcal{A}_{L, \delta}^{(4)}\right)=-v\left(\frac{d}{2}-1\right)
$$

For $v<0$ and all $\lambda \geq \lambda^{*}$, we have instead,

$$
\limsup _{L \rightarrow \infty} \frac{1}{L^{d}} \log P_{\lambda, L}^{m f}\left(\sum_{j=1}^{j(L)} j n_{j} \leq\left[u^{*}-|v|\right] L^{d}\right) \leq-c(v)
$$


where $c(v)>0$ and vanishes as $v \rightarrow 0$ as

$$
c(v) \sim \begin{cases}v^{2} & d \geq 5 \\ \frac{v^{2}}{|\log | v||} & d=4 \\ |v|^{3} & d=3\end{cases}
$$

Proof. See Theorem E.3 and Appendix H

\section{Long loops and Bose condensation.}

In this section we show that, in the mean field model, the excess density concentration $\rho-\rho^{*}$ on large loops implies the phenomenon of condensation (i.e. a finite fraction of the number of particles occupies the state of zero momentum ).

The reduced density matrices (RDM) are the quantum analogue of correlation functions $[\mathbf{1}][\mathbf{1 2}]$ and the Fourier transform of the one point RDM, in the case of periodic boundary conditions with translational invariant potentials, gives (Onsager Penrose [11]) the average number of particles of momentum $2 \pi p / L, p \in \mathbb{Z}^{d}$ :

$$
\hat{\rho}_{\Lambda}(p)=\int_{\Lambda} \rho_{\Lambda}(0, z) e^{i \frac{2 \pi p}{L} z} d z
$$

Using the language of loops, in the mean field case, where the interaction does not depend on the position of the particles, the one point RDM reads [5]:

$$
\rho_{\Lambda}^{m f}(x, y)=\sum_{j_{0}} \Xi_{\lambda, L}^{-1} \sum_{\underline{n}} w(\underline{n}) e^{-\beta \frac{\left(\sum_{j} j\left(n_{j}+\delta_{j, j_{0}}\right)\right)^{2}}{2 L^{d}}} e^{\lambda \beta j_{0}} \sum_{k \in \mathbb{Z}^{d}} \frac{e^{-\frac{(k L+(x-y))^{2}}{2 \beta j_{0}}}}{\left(2 \pi \beta j_{0}\right)^{d / 2}}
$$


Theorem 6.1. For $d \geq 3$ and any $\beta$, when $\lambda$ is larger than $\lambda^{*}$

$$
\lim _{L \rightarrow \infty} \frac{\hat{\rho}_{\Lambda}^{m f}(0)}{L^{d}}=\lambda-\lambda^{*}
$$

Proof From (6.1) and (6.2) we get that:

$$
\begin{gathered}
\hat{\rho}_{\Lambda}^{m f}(0)=\int_{\Lambda} \rho_{\Lambda}^{m f}(0, z) d z=\sum_{j_{0}} \Xi_{\lambda, L}^{-1} \sum_{\underline{n}} w(\underline{n}) e^{-\beta \frac{\left(\sum_{j} j\left(n_{j}+\delta_{j, j_{0}}\right)\right)^{2}}{2 L^{d}}} e^{\lambda \beta \sum_{j} j\left(n_{j}+\delta_{j, j_{0}}\right)} \\
=E_{\lambda, L}^{m f}\left(\sum_{j} \frac{j n_{j}}{L^{d} a(\beta j, L)}\right)
\end{gathered}
$$

The Theorem is proved using (4.5) and (cfr. (D.62))

$$
0<a(\beta j, L)-\rho_{j} \leq C \rho_{j} e^{-L^{2} /(2 \beta j)}
$$

\section{Appendix A}

In this appendix we recall the relation between the usual definition of the canonical partition function for a free Bose gas and its representation in the loops language given in (2.4).

The canonical partition function for a system of $N$ identical bosons is

$$
Z_{N}=\operatorname{Tr} e^{-\beta H_{N}}
$$

where $H_{N}$ is the Hamiltonian operator and the trace involves only symmetrized states. 
Theorem A.1. Let $H_{N}=-\sum_{i=1}^{N} \Delta_{i}$ be the hamiltonian of $N$ free Bosons in a cubic box of size $L$ with periodic boundary conditions, then

$$
Z_{N, L}=\sum_{\underline{\nu}:|\underline{\mid}|=N} e^{-\beta \sum_{p}\left(\frac{2 \pi p}{L}\right)^{2} \nu_{p}}=\sum_{\underline{n}: \sum j n_{j}=N} \prod_{j} \frac{1}{n_{j} !}\left(\frac{\sum_{p} e^{-\beta j\left(\frac{2 \pi p}{L}\right)^{2}}}{j}\right)^{n_{j}}
$$

Proof. The Bosons states in the momentum representation can be written as $|\underline{\nu}>=| \nu_{p}, p \in \mathbb{Z}^{d}>, \nu_{p}$ being the number of Bosons with momentum equal to $k=2 \pi p L^{-1}$. The energy in such a state is equal to $\sum_{p} \epsilon_{p} \nu_{p}$, $\epsilon_{p}=\left(2 \pi p L^{-1}\right)^{2}$, hence the first equality in (A.1).

To prove the second one, let $\lambda<0$ and define:

$$
\mathcal{Z}(\lambda):=\sum_{N} e^{\beta \lambda N} \sum_{\underline{\nu}:|\underline{\underline{\nu}}|=N} e^{-\beta \sum_{p} \epsilon_{p} \nu_{p}}
$$

that can be rewritten as:

$$
\begin{aligned}
\mathcal{Z}(\lambda) & =\exp \left\{-\sum_{p} \ln \left(1-e^{-\beta\left(\epsilon_{p}-\lambda\right)}\right)\right\}=\exp \left\{\sum_{j}\left(\sum_{p} \frac{e^{-\beta\left(\epsilon_{p}-\lambda\right) j}}{j}\right)\right\} \\
& =\sum_{M} \frac{1}{M !}\left[\sum_{j}\left(\sum_{p} \frac{e^{-\beta\left(\epsilon_{p}-\lambda\right) j}}{j}\right)\right]^{M} \\
& =\sum_{\underline{n}} \prod_{j}\left[\sum_{p} \frac{e^{-\beta\left(\epsilon_{p}-\lambda\right) j}}{j}\right]^{n_{j}} \frac{1}{n_{j} !} \\
& =\sum_{N} e^{\beta \lambda N} \sum_{\underline{n}: \sum_{j} j n_{j}=N} \prod_{j}\left[\frac{\sum_{p} e^{-\beta j \epsilon_{p}}}{j}\right]^{n_{j}} \frac{1}{n_{j} !}
\end{aligned}
$$

Since $\mathcal{Z}(\lambda)$ is analytic in $\lambda$ for $\operatorname{Re} \lambda<0$, (A.1) follows. 
An alternative proof working in the configuration representation can be obtained as follows.

$$
Z_{N, L}=\frac{1}{N !} \sum_{\pi} \int d r_{1} \ldots d r_{N}\left\langle r_{\pi_{1}} \ldots r_{\pi_{N}}\left|e^{-\beta H_{N}}\right| r_{1} \ldots r_{N}\right\rangle
$$

where $\sum_{\pi}$ is the sum over all permutations of $(1,2, \ldots, N)$. Since any permutation breaks up into cycles (loops), we have

$$
Z_{N}=\frac{1}{N !} \sum_{n_{1}, n_{2}, \ldots} c\left(n_{1}, n_{2}, \ldots\right) \prod_{j} Z^{n_{j}}
$$

where

a) $c\left(n_{1}, n_{2}, \ldots\right)=N ! \prod_{j} \frac{1}{j^{n_{j}}} \frac{1}{n_{j} !}$ is the number of ways of having $n_{1}$ loops of length $1, n_{2}$ of length 2 , etc.

b) the sum is over all combinations of permutations s.t. $\sum j n_{j}=N$

c) $Z(j)=\sum_{p} e^{-\beta j \epsilon_{p}}$, where $-\epsilon_{p}$ are the eigenvalues of the Laplace operator $\Delta$.

In the case of a free Bose gas in a cubic box of size $L$ with periodic boundary conditions

$$
Z_{N, L}=\sum_{\underline{n}: \sum j n_{j}=N} \prod_{j} \frac{1}{n_{j} !}\left(\frac{\sum_{p} e^{-\beta j\left(\frac{2 \pi p}{L}\right)^{2}}}{j}\right)^{n_{j}}
$$

thus deriving again the last equality in (A.1).

Finally, to justify equation (2.3), we prove the following lemma.

Lemma A.2. For any $L$ and $\alpha>0$

$$
L^{d} \sum_{k \in \mathbb{Z}^{d}} \frac{e^{-(k L)^{2} /(2 \alpha)}}{(2 \pi \alpha)^{d / 2}}=\sum_{k \in \mathbb{Z}^{d}} e^{-\frac{1}{2} \alpha(2 \pi k / L)^{2}}
$$


Proof. Equation (A.4) follows from the identities

$$
e^{-(k L)^{2} /(2 \alpha)}=\frac{1}{(2 \pi)^{d / 2}} \int_{\mathbb{R}^{d}} e^{-x^{2} / 2+i L k x / \sqrt{\alpha}} d x
$$

and

$$
\frac{1}{(2 \pi)^{d}} \sum_{k \in \mathbb{Z}^{d}} e^{i v k}=\sum_{k \in \mathbb{Z}^{d}} \delta(v-2 \pi k)
$$

\section{Appendix B}

The canonical partition function (A.1) of the free Bose gas can be written as

$$
\begin{gathered}
Z_{N, L}=\sum_{\left\{n_{\underline{k}}\right\}} \mathbf{1}_{\sum n_{\underline{k}}=N} e^{-\beta \sum_{\underline{k}} n_{\underline{k}} E_{\underline{k}}}=\sum_{M=0}^{N} \tilde{Z}_{M, L} \\
\tilde{Z}_{M, L}=\sum_{\left\{n_{\underline{k}}, \underline{k} \neq 0\right\}} \mathbf{1}_{\sum_{\underline{k} \neq 0} n_{\underline{k}}=M} e^{-\beta \sum_{\underline{k} \neq 0} n_{\underline{k}} E_{\underline{k}}}
\end{gathered}
$$

where the momentum $\underline{k}$ takes values in the set $\left\{2 \pi \underline{n} / L, \underline{n} \in \mathbb{Z}^{d}\right\}, n_{\underline{k}} \in \mathbb{Z}$ and $E_{\underline{k}}=\underline{k}^{2} / 2$.

In this appendix we study the tail properties of the probability distribution on $\mathbb{N}$ with density

$$
P_{L}(M)=\frac{\tilde{Z}_{M, L}}{Q_{L}} \quad, \quad Q_{L}=\sum_{M=0}^{\infty} \tilde{Z}_{M, L}
$$

and mean value

$$
<M>_{L}=\sum_{\underline{k} \neq 0} \frac{1}{e^{\beta E_{\underline{k}}}-1} \equiv L^{d} \lambda_{L}^{*}
$$

We remark that this probability distribution is the canonical distribution of the total number of particles with $k \neq 0$ for a free Bose gas. These results 
will be used in the sequel to prove small and large deviation both in the free and mean field case.

We want to study the asymptotic properties of the probability measure $P_{L}$ as $L \rightarrow \infty$. To begin with, note that, if $d \geq 3, \lim _{L \rightarrow \infty} \lambda_{L}^{*}$ does exist and

$$
\lambda^{*}=\lim _{L \rightarrow \infty} \lambda_{L}^{*}=\int_{\mathbb{R}^{d}} \frac{d \underline{k}}{(2 \pi)^{d}} \frac{1}{e^{\beta E_{\underline{k}}}-1}
$$

Let us define

$$
c_{L}=L^{d-2}\left(\lambda^{*}-\lambda_{L}^{*}\right)
$$

Lemma B.1. For any $d \geq 3, c_{L}$ has a limit as $L \rightarrow \infty$ and

$$
c^{*} \equiv \lim _{L \rightarrow \infty} c_{L}=\int_{0}^{\infty} d t\left[1-\sum_{\underline{n} \neq 0} \frac{e^{-\underline{n}^{2} /(2 \beta t)}}{(2 \pi \beta t)^{d / 2}}\right]
$$

Proof. (3.2) and (B.4) imply that

$$
L^{d} \lambda_{L}^{*}=\sum_{\underline{k} \neq 0} \sum_{j=1}^{\infty} e^{-\beta j E_{\underline{k}}}=\sum_{j=1}^{\infty}\left[L^{d} a(\beta j, L)-1\right]
$$

while (B.5) implies that

$$
L^{d} \lambda^{*}=L^{d} \sum_{j=1}^{\infty} \frac{1}{(2 \pi \beta j)^{d / 2}}
$$

hence, by using the definition of $a(t, L)$ in $(3.2)$, we get

$$
c_{L}=\frac{1}{L^{2}} \sum_{j=1}^{\infty}\left[1-\sum_{\underline{n} \neq 0} \frac{e^{-\underline{n}^{2} /\left(2 \beta t_{j}\right)}}{\left(2 \pi \beta t_{j}\right)^{d / 2}}\right] \quad, \quad t_{j}=\frac{j}{L^{2}}
$$

The lemma follows from this expression, easily implying the convergence of the sum over $j \leq L^{2}$, and the identity, following from (3.2) (with $L=1$ ),

$$
1-\sum_{\underline{n} \neq 0} \frac{e^{-\underline{n}^{2} /(2 t)}}{(2 \pi t)^{d / 2}}=\frac{1}{(2 \pi t)^{d / 2}}-\sum_{\underline{n} \neq 0} e^{-2 \pi^{2} t \underline{n}^{2}}
$$


which implies immediately the convergence of the sum over $j \geq L^{2}$.

Let us now define

$$
y_{L}=\frac{M-L^{d} \lambda_{L}^{*}}{h_{L}} \quad, \quad h_{L}= \begin{cases}L^{2} & d=3 \\ L^{2} \sqrt{\log L} & d=4 \\ L^{d / 2} & d \geq 5\end{cases}
$$

An important role in this appendix has the following Theorem

Theorem B.2. If $M$ is a random variable with probability (B.3), the distribution function of the random variable $y_{L}$ converges, as $L \rightarrow \infty$, to the distribution function of a random variable $y$ on $\mathbb{R}$ with mean 0 and smooth density $\rho(y)$ strictly positive, whose Laplace transform $F(\sigma)=$ $\int d y \rho(y) \exp (-\sigma y), \sigma \in \mathbb{C}$, is given, if $d=3$ and $\Re \sigma>-2 \pi^{2} \beta$, by

$$
F(\sigma)=\exp \left(\sum_{\underline{n} \neq 0} G\left(\frac{\sigma}{2 \pi^{2} \beta \underline{n}^{2}}\right)\right) \quad, \quad G(u)=u-\log (1+u)
$$

while, if $d \geq 4$ and $\Re \sigma \in \mathbb{R}$,

$$
F(\sigma)=e^{\frac{1}{2} c_{0} \sigma^{2}} \quad, \quad c_{0}= \begin{cases}\frac{1}{2 \pi^{2} \beta^{2}} & d=4 \\ \frac{1}{(2 \pi \beta)^{d / 2}} \sum_{j=1}^{\infty} j^{1-d / 2} & d \geq 5\end{cases}
$$

Moreover, there exists a constant $C$, independent of $L$ and $M$, such that

$$
\left(1+y_{L}^{2}\right) h_{L} P_{L}(M) \leq C
$$

and, given any $y \in \mathbb{R}$, if we choose $M=M_{L}^{*}$ so that $y_{L}^{*}=\left(M_{L}^{*}-\right.$ $\left.L^{d} \lambda_{L}^{*}\right) / h_{L} \underset{L \rightarrow \infty}{\longrightarrow} y$, then

$$
h_{L} P_{L}\left(M_{L}^{*}\right) \underset{L \rightarrow \infty}{\longrightarrow} \rho(y)
$$

Proof. To begin with, we shall prove that the Laplace transform of $y_{L}, F_{L}(\sigma)=\sum_{M=0}^{\infty} P_{L}(M) \exp \left[-\sigma\left(M-L^{d} \lambda_{L}^{*}\right) / h_{L}\right]$, is well defined and convergent as $L \rightarrow \infty$, if $\Re \sigma \in\left(\sigma_{0}, \infty\right)$, where $\sigma_{0}=-2 \pi^{2} \beta$, if $d=3$, and 
$\sigma_{0}=-\infty$, if $d \geq 4$; this implies in particular that the characteristic function $f_{L}(t)=F_{L}(-i t), t \in \mathbb{R}$, is convergent for any $t$. By analyzing the decaying properties in $t$ of $f_{L}(t)$, we shall also prove the bound (B.15), implying that the distribution function of $y_{L}$ is convergent and that its limit is the distribution function of a probability measure on $\mathbb{R}$; in fact, by a simple application of dominated convergence Theorem,

$$
\sum_{0 \leq M \leq h_{L} y+L^{d} \lambda_{L}^{*}} P_{L}(M)=\frac{1}{h_{L}} \sum_{0 \leq M \leq h_{L} y+L^{d} \lambda_{L}^{*}} h_{L} P_{L}(M) \underset{L \rightarrow \infty}{\longrightarrow} \int_{-\infty}^{y} \rho(z) d z
$$

Note that this result follows from the convergence of $f_{L}(t)$ to $f(t)$, without using the bound (B.15), since $P_{L}(M)$ is a probability measure [3]. We are stressing here the role of (B.15) only because we shall generalize in the following the previous argument to some cases where $P_{L}(M)$ is not a probability measure, even if $\sum_{M=0}^{\infty} P_{L}(M)=1$.

Finally, by analyzing the properties of the limiting measure Laplace transform, we shall prove that this measure has a smooth and strictly positive density.

By a straightforward calculation, one can see that

$$
\begin{aligned}
& \log F_{L}(\sigma)=\frac{L^{d} \lambda_{L}^{*}}{h_{L}} \sigma-\sum_{\underline{k} \neq 0} \log \frac{1-e^{-\beta E_{\underline{\underline{k}}}-\sigma / h_{L}}}{1-e^{-\beta E_{\underline{k}}}}= \\
& =\sum_{\underline{k} \neq 0}\left[\frac{\sigma}{h_{L}} \frac{1}{e^{\beta E_{\underline{k}}}-1}-\log \left(1+\frac{1-e^{-\sigma / h_{L}}}{e^{\beta E_{\underline{k}}}-1}\right)\right]= \\
& =\sum_{\underline{k} \neq 0} \frac{1}{e^{\beta E_{\underline{k}}}-1}\left[\frac{\sigma}{h_{L}}-\left(1-e^{-\sigma / h_{L}}\right)\right]+\sum_{\underline{n} \neq 0} G\left(\frac{1-e^{-\sigma / h_{L}}}{e^{2 \pi^{2} \beta \underline{n^{2}} / L^{2}}-1}\right)
\end{aligned}
$$

where $G(u)=u-\log (1+u)$.

Let us consider first the case $d=3$. Then $h_{L}=L^{2}$, so that $F_{L}(\sigma)$ is well defined for $\Re \sigma>\sigma_{0} \equiv-2 \pi^{2} \beta$; hence we shall fix $\sigma$ so that this condition is satisfied. Then the first term in the third line of (B.18) goes to 0 as $L \rightarrow \infty$, 
since it is bounded by $C L^{-1}$, where $C$ (here and in the following) denotes a suitable positive constant, depending on $\sigma$ but independent of $L$. Note that

$$
u_{\underline{n}, L} \equiv \frac{1-e^{-\sigma / h_{L}}}{e^{2 \pi^{2} \beta \underline{n}^{2} / L^{2}}-1} \underset{L \rightarrow \infty}{\longrightarrow} u_{\underline{n}}^{*} \equiv \frac{\sigma}{2 \pi^{2} \beta \underline{n}^{2}}
$$

and that $\left|u_{\underline{n}, L}\right|,\left|u_{\underline{n}}^{*}\right| \leq C \underline{n}^{-2}$ and $\left|u_{\underline{n}, L}-u_{\underline{n}}^{*}\right| \leq C / L^{2}$. On the other hand, $\Re u_{\underline{n}, L}$ and $\Re u_{n}^{*}$ are larger of some constant $u_{0}>-1$, for any $\underline{n}$; since $G^{\prime}(u)=$ $u /(1+u)$, it immediately follows that

$$
\begin{aligned}
\left|G\left(u_{\underline{n}, L}\right)-G\left(u_{\underline{n}}^{*}\right)\right| & \leq C\left|u_{\underline{n}, L}-u_{\underline{n}}^{*}\right| \underline{n}^{-2} \leq C \underline{n}^{-7 / 2}\left|u_{\underline{n}, L}-u_{\underline{n}}^{*}\right|^{1 / 4} \leq \\
& \leq C|\underline{n}|^{-7 / 2} L^{-1 / 2}
\end{aligned}
$$

It follows that $\log F_{L}(\sigma)$ and $F_{L}(\sigma)$ are convergent for $L \rightarrow \infty$ and that, if $F(\sigma)=\lim _{L \rightarrow \infty} F_{L}(\sigma)$,

$$
\Pi(\sigma) \equiv \log F(\sigma)=\sum_{\underline{n} \neq 0} G\left(\frac{\sigma}{2 \pi^{2} \beta \underline{n}^{2}}\right)
$$

It is not hard to show that $\Pi(\sigma)$ is differentiable and that

$$
\Pi^{\prime}(\sigma)=\sum_{\underline{n} \neq 0} \frac{\sigma}{2 \pi^{2} \beta \underline{n}^{2}\left(\sigma+2 \pi^{2} \beta \underline{n}^{2}\right)}
$$

implying that, if $x \in \mathbb{R}$,

$$
\lim _{x \rightarrow+\infty} \Pi^{\prime}(x)=+\infty \quad, \quad \lim _{x \rightarrow \sigma_{0}^{+}} \Pi^{\prime}(x)=-\infty
$$

Let us now call $P(d y)$ the probability measure such that

$$
F(\sigma)=e^{\Pi(\sigma)}=\int P(d y) e^{-\sigma y}
$$

The property (B.23) easily implies that the support of $P(d x)$ is the full real line. Moreover, the characteristic function $f(t)$ of $P(d x)$ is given by the equation

$$
f(t)=e^{\Pi(-i t)}=\prod_{\underline{n} \neq 0} \frac{e^{-i t a \underline{n}^{-2}}}{1-i t a \underline{n}^{-2}} \quad, \quad a=\left(2 \pi^{2} \beta\right)^{-1}
$$


By using the bound $\log (1+x) \geq 2 x / 3$, valid for $0 \leq x \leq 1 / 2$, we get, if $|t| \geq 1$

$$
\begin{aligned}
& |f(t)| \leq \prod_{\underline{n} \neq 0}\left(1+t^{2} a^{2}|\underline{n}|^{-4}\right)^{-1 / 2} \leq \\
& \leq \prod_{|\underline{n}| \leq(\sqrt{2} a|t|)^{1 / 2}}(3 / 2)^{-1 / 2} \prod_{|\underline{n}|>(\sqrt{2} a|t|)^{1 / 2}} e^{-t^{2} a^{2}|\underline{n}|^{-4} / 3} \leq e^{-C|t|^{3 / 2}}
\end{aligned}
$$

This bound and the support properties of $P(d y)$ imply that $P(d y)=\rho(y) d y$, with $\rho(y)$ a strictly positive smooth function on $\mathbb{R}$.

In order to complete the proof of the Theorem in the case $d=3$, we still have to prove the strong convergence property (B.16), together with the uniform bound (B.15) on $h_{L} P_{L}(M)$. Note that the definition of characteristic function implies that

$$
h_{L} P_{L}(M)=\frac{1}{2 \pi} \int_{-\pi h_{L}}^{+\pi h_{L}} d t e^{-i t y_{L}} f_{L}(t)
$$

By using (B.18), we see that

$$
f_{L}(t)=F_{L}(-i t)=\prod_{\underline{n} \neq 0} \frac{e^{v_{n, L}}}{1+u_{n, L}}
$$

where $u_{n, L}$ is given by (B.19) with $\sigma=-i t$ and

$$
v_{n, L}=\frac{-i t}{L^{2}\left(e^{2 \pi^{2} \beta \underline{n}^{2} / L^{2}}-1\right)}
$$

It follows that $\left|f_{L}(t)\right| \leq \prod_{n \neq 0}\left|1+u_{n, L}\right|^{-1}$. Moreover, by using (B.19), we see that, if $|t| \leq \pi L^{2} / 2$ and $|n|^{2} \leq|t|,\left|1+u_{n, L}\right| \geq 1+\delta$, with a suitable $\delta>0$. Hence, if $|t| \leq \pi L^{2} / 2,\left|f_{L}(t)\right| \leq \prod_{0<|\underline{n}| \leq|t|^{1 / 2}}(1+\delta)^{-1} \leq \exp \left(-C|t|^{-3 / 2}\right)$. If $\pi L^{2} / 2 \leq|t| \leq \pi L^{2}$, the same result is obtained, by observing that in this case, if $|n| \leq L,\left|1+u_{n, L}\right| \geq 1+1 /\left(e^{2 \pi^{2} \beta}-1\right)$, so that $\left|f_{L}(t)\right| \leq \exp \left(-C L^{3}\right) \leq$ $\exp \left(-C|t|^{-3 / 2}\right)$. Hence, we can show that, uniformly in $L$,

$$
\left|f_{L}(t)\right| \leq \exp \left(-C|t|^{3 / 2}\right) \quad, \quad|t| \leq \pi h_{L}
$$


which implies, together with (B.27), that $h_{L} P_{L}(M) \leq C$, with $C$ independent of $L$ and $M$. Moreover, by the dominated Lebesgue convergence Theorem, we get, for any $y \in \mathbb{R}$,

$$
y_{L} \underset{L \rightarrow \infty}{\longrightarrow} y \quad \Rightarrow \quad h_{L} P_{L}(M) \underset{L \rightarrow \infty}{\longrightarrow} \frac{1}{2 \pi} \int_{-\infty}^{+\infty} e^{i t y} f(t)=\rho(y)
$$

In order to complete the proof of (B.15), we use the identity

$$
\left(-i y_{L}\right)^{2} h_{L} P_{L}(M)=\frac{1}{2 \pi} \int_{-\pi h_{L}}^{+\pi h_{L}} e^{-i t y_{L}} f_{L}^{\prime \prime}(t)
$$

Since $f_{L}^{\prime \prime}(t)=f_{L}(t)\left[\Pi_{L}^{\prime}(-i t)^{2}+\Pi_{L}^{\prime \prime}(-i t)\right]$, where $\Pi_{L}(-i t)=\log F_{L}(-i t)$, and, as one can check easily by proceeding as in the analysis given before of $\log F_{L}(\sigma)$, uniformly in $L$,

$$
\left|\Pi_{L}^{\prime}(-i t)\right| \leq C|t| \quad, \quad\left|\Pi_{L}(-i t)\right| \leq C
$$

the bound (B.15) immediately follows from the bound (B.30).

Let us now suppose that $d=4$. Then $h_{L}=L^{2} \sqrt{\log L}$, so that, given any $x<0, F_{L}(\sigma)$ is well defined for $\Re \sigma>x$, if $L>\exp \left(-x /\left(2 \pi^{2} \beta\right)\right.$. Moreover, as in the case $d=3$, the first term in the third line of (B.18) goes to 0 as $L \rightarrow \infty$, since it is bounded by $C(\log L)^{-1 / 2}$.

If we define $u_{\underline{n}, L}$ as in (B.19), $\left|u_{\underline{n}, L}\right| \leq C\left(\underline{n}^{2} \sqrt{\log L}\right)^{-1}$, with $C$ only depending on $\sigma$ if $L$ is large enough. Hence, if $\tilde{G}(u)=u-\log (1+u)-u^{2} / 2$,

$$
\left|\sum_{\underline{n} \neq 0} \tilde{G}\left(u_{\underline{n}, L}\right)\right| \leq \frac{C}{(\log L)^{3 / 2}} \sum_{\underline{n} \neq 0} \frac{1}{|\underline{n}|^{6}} \underset{L \rightarrow \infty}{\longrightarrow} 0
$$


Note also that $L^{-4} \sum_{|\underline{n}| \geq L}\left[\exp \left(a \underline{n}^{2} / L^{2}\right)-1\right]^{-2}$ is bounded for $L \rightarrow \infty$, for any $a>0$, and that

$$
\begin{aligned}
& \frac{1}{\log L} \sum_{0<|\underline{n}| \leq L}\left[\frac{1}{\left(a \underline{n}^{2}\right)^{2}}-\frac{1}{L^{4}\left(e^{a \underline{n}^{2} / L^{2}}-1\right)^{2}}\right] \leq \\
& \frac{C}{L^{2} \log L} \sum_{0<|\underline{n}| \leq L} \frac{1}{|\underline{n}|^{4}} \underset{L \rightarrow \infty}{\longrightarrow} 0
\end{aligned}
$$

so that $(\log L)^{-1} L^{-4} \sum_{\underline{n} \neq 0}\left[\exp \left(a \underline{n}^{2} / L^{2}\right)-1\right]^{-2}$ is convergent for $L \rightarrow \infty$ and

$$
\begin{aligned}
c_{0} & =\lim _{L \rightarrow \infty} \frac{1}{\log L} \sum_{\underline{n} \neq 0} \frac{1}{L^{4}\left(e^{a \underline{n}^{2} / L^{2}}-1\right)^{2}}=\lim _{L \rightarrow \infty} \frac{1}{\log L} \sum_{0<|\underline{n}| \leq L} \frac{1}{a^{2}|\underline{n}|^{4}}= \\
& =\frac{1}{a^{2}} \lim _{L \rightarrow \infty} \frac{1}{\log L} \int_{1 \leq|\underline{x}| \leq L} \frac{d^{4} \underline{x}}{|\underline{x}|^{4}}=\frac{2 \pi^{2}}{a^{2}}
\end{aligned}
$$

$\mathrm{Bu}$ using (B.18) and (B.36) with $a=2 \pi^{2} \beta$, it is now easy to prove that $\log F_{L}(\sigma)$ is convergent for $L \rightarrow \infty$ and that

$$
\begin{aligned}
& \Pi(\sigma)=\lim _{L \rightarrow \infty} \log F_{L}(\sigma)= \\
& =\frac{1}{2} \sigma^{2} \lim _{L \rightarrow \infty} \frac{1}{L^{4} \log L} \sum_{\underline{n} \neq 0} \frac{1}{\left(e^{2 \pi^{2} \beta \underline{n}^{2} / L^{2}}-1\right)^{2}}=\frac{1}{2} \frac{2}{(2 \pi \beta)^{2}} \sigma^{2}
\end{aligned}
$$

It follows immediately, if we define $P(d y)$ as in the case $d=3$, that $P(d y)$ is a Gaussian probability measure with density $\rho(y)=\left(2 \pi c_{0}\right)^{-1 / 2} \exp \left[-y^{2} /\left(2 c_{0}\right)\right]$, with $c_{0}=2 /(2 \pi \beta)^{2}$. The proof of (B.15) and (B.16) in the case $d=3$ can be easily extended to this case; we omit the details.

Let us finally consider the case $d \geq 5$. Then $h_{L}=L^{d / 2}$ and we can proceed as in the previous case, the only relevant difference being that now $\Pi(\sigma)$ gets a contribution also from the first term in the third line of (B.18). 
We find that $\Pi(\sigma)=\frac{1}{2} c_{0} \sigma^{2}$, with

$$
\begin{aligned}
& c_{0}=\lim _{L \rightarrow \infty} \frac{1}{L^{d}} \sum_{\underline{n} \neq 0}\left[\frac{1}{e^{2 \pi^{2} \beta \underline{n}^{2} / L^{2}}-1}+\frac{1}{\left(e^{2 \pi^{2} \beta \underline{n}^{2} / L^{2}}-1\right)^{2}}\right]= \\
& \int \frac{d^{d} \underline{k}}{(2 \pi)^{d}} \frac{e^{-\beta E_{\underline{k}}}}{\left(1-e^{\left.-\beta E_{\underline{k}}\right)^{2}}\right.}=\sum_{j_{1}, j_{2} \geq 0} \int \frac{d^{d} \underline{k}}{(2 \pi)^{d}} e^{-\beta E_{\underline{k}}\left(j_{1}+j_{2}+1\right)}=\sum_{j \geq 1} \frac{j}{(2 \pi \beta j)^{d / 2}}
\end{aligned}
$$

The proof of (B.15) and (B.16) in the case $d=3$ can be easily extended also to this case, so completing the proof of the Theorem.

\section{Appendix C. Proof of Theorem 3.1}

Note that the mean field grand canonical partition function can be written as

$$
\Xi_{\lambda, L}^{m f}=e^{\frac{1}{2} \beta \lambda^{2} L^{d}} \sum_{N=0}^{\infty} e^{-\frac{\beta}{2} L^{d}\left(\frac{N}{L^{d}}-\lambda\right)^{2}} Z_{N, L}
$$

and that

$$
\begin{aligned}
P_{L}(M \leq N) & =P_{L}\left(y_{L} \leq \frac{L^{d}}{h_{L}}\left(\lambda-\lambda^{*}\right)+\frac{L^{d / 2}}{h_{L}} x_{N}+\frac{L^{2}}{h_{L}} c_{L}\right) \\
x_{N} & =L^{d / 2}\left(\frac{N}{L^{d}}-\lambda\right)
\end{aligned}
$$

Let us now define

$$
\Gamma_{\lambda, L} \equiv \frac{\Xi_{\lambda, L}^{m f}}{e^{\frac{1}{2} \beta \lambda^{2} L^{d}} Q_{L} L^{d / 2}}=\frac{1}{L^{d / 2}} \sum_{N=0}^{\infty} e^{-\frac{\beta}{2} L^{d}\left(\frac{N}{L^{d}}-\lambda\right)^{2}} P_{L}(M \leq N)
$$

Theorem 3.1 follows from the following Lemma. 
Lemma C.1. If $\lambda \geq \lambda^{*}$, the quantity $\Gamma_{\lambda, L}$ has a limit as $L \rightarrow \infty$. If $\lambda>\lambda^{*}$, we have, for any $d \geq 3$,

$$
\lim _{L \rightarrow \infty} \Gamma_{\lambda, L}=\sqrt{\frac{2 \pi}{\beta}}
$$

while, if $\lambda=\lambda_{*}$, we have

$$
0<\lim _{L \rightarrow \infty} \Gamma_{\lambda, L}= \begin{cases}\sqrt{\frac{2 \pi}{\beta}} \int_{-\infty}^{c^{*}} d y \rho(y) & d=3 \\ \sqrt{\frac{\pi}{2 \beta}} & d=4 \\ \int_{-\infty}^{+\infty} d x e^{-\beta x^{2} / 2} \int_{-\infty}^{x} d y \rho(y) & d \geq 5\end{cases}
$$

where $\rho(y)$ is the density probability defined in Theorem B.2.

Moreover, if $N_{L}=\left[u L^{d}\right]$ ([.] denotes the integer part), $u>\lambda^{*}$, and $N_{L}^{*}=\left[\lambda^{*} L^{d}\right]$, then

$$
\lim _{L \rightarrow \infty} \frac{Z_{N_{L}, L}}{Z_{N_{L}^{*}, L}}= \begin{cases}1 / P\left(y \leq c_{*}\right) & d=3 \\ 2 & d \geq 4\end{cases}
$$

Proof. By theorem B.2, $P_{L}\left(y_{L} \leq \bar{y}\right) \underset{L \rightarrow \infty}{\longrightarrow} \int_{0}^{\bar{y}} d y \rho(y)$, for any fixed $\bar{y}, \rho(y)$ being a strictly positive function depending on the dimension $d$. Hence, by using (C.2) and Lemma B.1, we can easily show that, if $\lambda>\lambda^{*}$ and $x_{N} \underset{L \rightarrow \infty}{\longrightarrow} x$,

$$
P_{L}(M \leq N) \underset{L \rightarrow \infty}{\longrightarrow} 1 \quad, \quad \forall x
$$

while, if $\lambda=\lambda^{*}$ and $x_{N} \underset{L \rightarrow \infty}{\longrightarrow} x$,

$$
P_{L}(M \leq N) \underset{L \rightarrow \infty}{\longrightarrow} \begin{cases}P\left(y \leq c_{*}\right) & d=3 \\ P(y \leq 0)=1 / 2 & d=4 \\ P(y \leq x) & d \geq 5\end{cases}
$$

Then (C.4) and (C.5) follow from (C.3) and a simple application of the dominated Lebesgue convergence theorem. The proof of (C.6) is a simple 
consequence of (C.2), (C.8) and the equation, valid if $N_{L}=\left[u L^{d}\right], u>\lambda^{*}$, and $N_{L}^{*}=\left[\lambda^{*} L^{d}\right]$

$$
\lim _{L \rightarrow \infty} \frac{Z_{N_{L}, L}}{Z_{N_{L}^{*}, L}^{*}}=\lim _{L \rightarrow \infty} \frac{P_{L}\left(M \leq N_{L}\right)}{P_{L}\left(M \leq N_{L}^{*}\right)}=\lim _{L \rightarrow \infty} \frac{P_{L}\left(y_{L} \leq \frac{L^{d}}{h_{L}}\left(u-\lambda^{*}\right)\right)}{P_{L}\left(y_{L} \leq c_{L} L^{2} / h_{L}\right)}
$$

By similar arguments, one can prove the following Theorem (see also [2] for the case $\lambda>\lambda^{*}$ ).

Theorem C.2. If $\lambda>\lambda^{*}$, the distribution of the random variable $x_{N}$ converges, as $L \rightarrow \infty$, to a Gaussian distribution with density $\exp \left(-\beta x^{2} / 2\right)$; the same result is true if $\lambda=\lambda^{*}$ and $d=3,4$. However, if $d>4$, the limiting distribution is still well defined, but it is not Gaussian anymore; it is proportional to $e^{-\beta x^{2} / 2} \int_{-\infty}^{x} d y \rho(y), \rho(y)$ being the density probability defined in Theorem B.2.

\section{Appendix D. Distribution of "short loops"}

In this appendix we will restrict to $d \geq 3$ and $\lambda \geq \lambda^{*}$ and study the distribution of the variables

$$
y_{A, L}=\frac{\sum_{j \in A} j n_{j}-L^{d} \rho_{A, L}}{h_{A, L}} \quad, \quad \rho_{A, L}=\sum_{j \in A} \rho_{j, L}
$$

where $A$ is a finite subset of $\mathbb{N}^{+}, L^{D} \rho_{j, L}$ is the mean value of $j n_{j}$ with respect to the mean field measure and $h_{A, L}$ is a suitable scaling factor. The main results are stated in Theorems D.4 and D.5 below, the main ingredient in the proofs is the reduction to the analysis of the probability distribution $P_{L}(M)$ defined in Appendix B. 
We start by deriving the following expression for $\rho_{j, L}$ :

$$
\rho_{j, L}=a(\beta j, L) \alpha_{j, L}
$$

where

$$
\alpha_{j, L}=\frac{1}{\Gamma_{\lambda, L}} \frac{1}{L^{d / 2}} \sum_{N \geq 0} e^{-\beta x_{N}^{2} / 2} P_{L}(M \leq N-j)
$$

with $x_{N}$ as in (C.2).

Proof of (D.2) - Let $w^{0}\left(j, n_{j}\right)$ be as in (2.1) with $\lambda=0$, then:

$$
\begin{aligned}
\rho_{j^{*}, L} & =\frac{e^{\frac{\beta \lambda^{2}}{2} L^{d}}}{\Xi_{\lambda, L}^{m f}} \sum_{N} e^{-\beta \frac{x_{N}^{2}}{2}} \sum_{\underline{n}: \sum_{j} j n_{j}=N} \prod_{j} w^{0}\left(j, n_{j}\right) \frac{j^{*} n_{j^{*}}}{L^{d}} \\
& =\frac{e^{\frac{\beta \lambda^{2}}{2} L^{d}}}{\Xi_{\lambda, L}^{m f}} a\left(\beta j^{*}, L\right) \sum_{N} e^{-\beta \frac{x_{N}^{2}}{2}} Z_{N-j^{*}, L}
\end{aligned}
$$

By (B.1) and (B.3) we get:

$$
\rho_{j^{*}, L}=\frac{e^{\frac{\beta \lambda^{2}}{2} L^{d}}}{\Xi_{\lambda, L}^{m f}} a\left(\beta j^{*}, L\right) Q_{L} \sum_{N} e^{-\beta \frac{x_{N}^{2}}{2}} P_{L}\left(M<N-j^{*}\right)
$$

hence (D.2) follows by (C.3).

Lemma D.1. For any $\lambda \geq \lambda^{*}$, there is a constant $C$, independent of $L$ and $j$, such that, if $h_{L}$ is defined as in (B.12),

$$
0<1-\alpha_{j, L} \leq C \frac{j}{h_{L}}
$$

Moreover, if $\lambda=\lambda^{*}$,

$$
\lim _{L \rightarrow \infty} \frac{h_{L}}{j}\left(1-\alpha_{j, L}\right)= \begin{cases}\frac{\rho\left(c^{*}\right)}{\int_{-\infty}^{c^{*}} d y \rho(y)} & d=3 \\ 2 \rho(0) & d=4 \\ \frac{\int_{-\infty}^{+\infty} d x e^{-\beta x^{2} / 2} \rho(x)}{\int_{-\infty}^{+\infty} d x e^{-\beta x^{2} / 2} \int_{-\infty}^{x} d y \rho(y)} & d \geq 5\end{cases}
$$

where $\rho(y)$ is defined as in Lemma C.1. 
Proof. Note that

$$
1-\alpha_{j, L}=\frac{1}{\Gamma_{\lambda, L}} \frac{1}{L^{d / 2}} \sum_{N \geq 0} e^{-\beta x_{N}^{2} / 2} P_{L}(N-j<M \leq N)
$$

By using the claim in Lemma C.1 that $h_{L} P_{L}(M)$ is bounded uniformly in $L$ and $M$, we get

$$
P_{L}(N-j<M \leq N)=\frac{1}{h_{L}} \sum_{M=N-j+1}^{N} h_{L} P_{L}(M) \leq C \frac{j}{h_{L}}
$$

which immediately implies (D.6), by using Lemma C.1. On the other hand, if $\lambda=\lambda^{*}$ and $M=N-r, r \geq 1$, the corresponding $y_{L}$ variable is equal, see (C.2), to $\left(L^{d / 2} x_{N}+L^{2} c_{L}-r\right) / h_{L}$, so that, by using (B.16),

$$
h_{L} P_{L}(M=N-r) \underset{L \rightarrow \infty}{\longrightarrow} \begin{cases}\rho\left(c^{*}\right) & d=3 \\ \rho(0) & d=4 \\ \rho(x) & d \geq 5\end{cases}
$$

(D.7) then follows from Lemma C.1 and dominated convergence Theorem.

If $\lambda>\lambda^{*}, h_{L} P_{L}(M=N-r)$ goes to 0 as $L \rightarrow \infty$, so we expect the bound (D.6) can be improved. This is especially true if $j$ is taken as a diverging function of $L$; in particular, if $j>\left(\lambda-\lambda^{*}\right) L^{d}$, it is easy to see that $\alpha_{j, L} \rightarrow 0$ as $L \rightarrow \infty$. In order to get good bounds in all these cases, we shall use the following large deviation bound for the probability measure $P_{L}(M)$.

Lemma D.2. Let $0<u_{1}<u_{2}$; then there exist $\bar{L}\left(u_{1}\right)$, such that the probabilities

$$
\begin{aligned}
& S_{L}^{+}\left(u_{1}, u_{2}\right) \equiv P_{L}\left(L^{d} \lambda_{L}^{*}+L^{d} u_{1} \leq M \leq L^{d} \lambda_{L}^{*}+L^{d} u_{2}\right) \\
& S_{L}^{-}\left(u_{1}, u_{2}\right) \equiv P_{L}\left(L^{d} \lambda_{L}^{*}-L^{d} u_{2} \leq M \leq L^{d} \lambda_{L}^{*}-L^{d} u_{1}\right)
\end{aligned}
$$


satisfy, for $L \geq \bar{L}\left(u_{1}\right)$, the following bounds.

$$
\begin{gathered}
e^{-a_{1} u_{2} L^{d-2}\left(1+\delta_{L}\right)} \leq S_{L}^{+}\left(u_{1}, u_{2}\right) \leq e^{-a_{1} u_{1} L^{d-2}\left(1-\delta_{L}\right)} \quad, \quad \forall u_{1}>0 \\
e^{-f\left(u_{2}\right)\left(L^{d} / h_{L}\right)^{2}\left(1+\delta_{L}\right)} \leq S_{L}^{-}\left(u_{1}, u_{2}\right) \leq e^{-f\left(u_{1}\right)\left(L^{d} / h_{L}\right)^{2}\left(1-\delta_{L}\right)} \quad, \quad u_{2}<\lambda^{*}
\end{gathered}
$$

where $\delta_{L}$ is a function which goes to 0 as $L \rightarrow \infty, a_{1}$ is a positive constant, depending on $d$, and $f(u)$ is a positive function of order $u^{2}$ for $u \rightarrow 0$ (equal indeed to $a_{2} u^{2}$ for $\left.d=2,3\right)$.

Proof. By (C.2), we can write

$$
S_{L}^{+}\left(u_{1}, u_{2}\right)=\int_{u_{1} L^{d} / h_{L}}^{u_{2} L^{d} / h_{L}} P_{L}(d y)=e^{\Pi_{L}(t)} \int_{u_{1} L^{d} / h_{L}}^{u_{2} L^{d} / h_{L}} e^{t y} P_{t, L}(d y)
$$

where $\Pi_{L}(t)=\log F_{L}(t), F_{L}(t)$ is the Laplace transform of $P_{L}(d y)$ given by (B.18), $t$ is any real number such that $F_{L}(t)$ is well defined and $P_{t, L}(d y)$ is the probability measure

$$
P_{t, L}(d y)=\frac{e^{-t y} P_{L}(d y)}{F_{L}(t)}
$$

By looking at (B.18), we see that $F_{L}(t)$ is defined for $t>t_{L}^{*}$, where $t_{L}^{*}$ is the value of $t$ such that the argument $u_{n, L}$ of the function $G(u)=u-\log (1+u)$ is equal to -1 if $|\underline{n}|=1$, that is $t_{L}^{*}=-a h_{L} / L^{2}$. We choose $t$ so that

$$
-\Pi_{L}^{\prime}(t)=\int d y y P_{t, L}(d y)=v \frac{L^{d}}{h_{L}} \quad, \quad v=\frac{u_{1}+u_{2}}{2}
$$

By using (B.18), this condition can be written

$$
\frac{1}{h_{L}} \sum_{\underline{n} \neq 0} \frac{\left(e^{-t / h_{L}}-1\right) e^{a \underline{n}^{2} / L^{2}}}{\left(e^{a \underline{n}^{2} / L^{2}}-e^{-t / h_{L}}\right)\left(e^{a \underline{n}^{2} / L^{2}}-1\right)}=v \frac{L^{d}}{h_{L}} \quad, \quad a=2 \pi^{2} \beta
$$

By proceeding as in the proof of Theorem B.2, it is easy to see that the sum in the l.h.s. is bounded by $C|t|$, if we extract from it the terms with 
$|\underline{n}|=1$; hence we get

$$
c_{L}-d \frac{t}{h_{L}^{2}} \frac{1}{a / L^{2}+t / h_{L}} \frac{L^{2}}{a}\left(1+\delta_{1, L}\right)=v \frac{L^{d}}{h_{L}}
$$

with $\delta_{1, L} \rightarrow 0$ and $c_{L} \rightarrow c$ as $L \rightarrow \infty$. It follows that

$$
t \frac{L^{d}}{h_{L}}=-a L^{d-2}\left(1+\delta_{2, L}\right)
$$

with $\delta_{2, L} \rightarrow 0$ as $L \rightarrow \infty$. It is easy to see that, for such a value of $t, \Pi_{L}(t)$ diverges as $C \log L$ for $d=3,4$ and as $L^{d-4}$ for $d \geq 5$, so that we can write

$$
S_{L}^{+}\left(u_{1}, u_{2}\right)=e^{-a v L^{d-2}\left(1+\delta_{L}\right)} \int_{u_{1} L^{d} / h_{L}}^{u_{2} L^{d} / h_{L}} e^{t\left(y-v L^{d} / h_{L}\right)} P_{t, L}(d y)
$$

with $\delta_{L} \rightarrow 0$ as $L \rightarrow \infty$. The upper bound in (D.13) easily follows from this equation. In order to prove the lower bound we have also to show that

$$
\int_{u_{1} L^{d} / h_{L}}^{u_{2} L^{d} / h_{L}} P_{t, L}(d y) \geq 1-\delta_{L}
$$

with $\delta_{L} \rightarrow 0$. This result can be deduced as the other ones from the properties of the Laplace transform of the measure $P_{t, L}(d y)$; we omit the details.

Let us now consider the upper bound of (D.14). We proceed as before, by writing

$$
\begin{aligned}
S_{L}^{-}\left(u_{1}, u_{2}\right) & =e^{\Pi_{L}(t)-t \Pi_{L}^{\prime}(t)} \int_{-u_{2} L^{d} / h_{L}}^{-u_{1} L^{d} / h_{L}} e^{t\left(y+u_{1} L^{d} / h_{L}\right)} P_{t, L}(d y) \leq \\
& \leq e^{\Pi_{L}(t)-t \Pi_{L}^{\prime}(t)}
\end{aligned}
$$

where $t$ is chosen so that $\Pi_{L}^{\prime}(t)=u_{1} L^{d} / h_{L}$. It is easy to see that $\Pi_{L}^{\prime}(t)$ is a monotone function and that $\lim _{t \rightarrow \infty} \Pi_{L}^{\prime}(t)=\lambda_{L}^{*} L^{d} / h_{L}$, so that $t$ is well defined for $L$ large enough, if $u_{1}<\lambda^{*}$. It turns out that $\lim _{L \rightarrow \infty} t\left(h_{L} / L^{d}\right)=$ $f_{0}(u)>0$, with $f_{0}(u)$ of order $u$ for $u \rightarrow 0$ (and equal to $c_{d} u$ for $d=3,4$, 
for some $\left.c_{d}>0\right)$. Moreover

$$
\Pi_{L}(t)-t \Pi_{L}^{\prime}(t)=-\int_{0}^{t} d s \int_{0}^{s} d u \Pi_{L}^{\prime \prime}(u) \quad, \quad C / 2<\Pi_{L}^{\prime \prime}(u) \leq C
$$

and one can prove that $\lim _{L \rightarrow \infty} \Pi_{l}^{\prime \prime}(u)=C_{d}>0$, uniformly for $0 \leq u \leq t$; this allows us to get the upper bound in (D.14). The lower bound is obtained in a similar way, by choosing $t$ so that $\Pi_{L}^{\prime}(t)=u_{2} L^{d} / h_{L}$ and by proving that $\int_{-u_{2} L^{d} / h_{L}}^{-u_{L}{ }^{d} / h_{L}} P_{t, L}(d y) \rightarrow 1 / 2$ for $L \rightarrow \infty$.

We can now prove the following bounds on the factors $\alpha_{j, L}$.

Lemma D.3. Given $d \geq 3, \lambda>\lambda^{*}$ and a sequence $j_{L}$ such that

$$
\lim _{L \rightarrow \infty} j_{L} / L^{d}=\gamma<\left(\lambda-\lambda^{*}\right)
$$

there exists $\bar{L}$ such that, if $L \geq \bar{L}$ and $j \leq j_{L}$,

$$
1-\alpha_{j, L} \leq C e^{-a_{3}\left(\lambda-\lambda^{*}-\gamma\right) L^{d-2}}
$$

where $C$ and $a_{3}$ are constants independent of $L$ and of $j$.

Moreover, if $\lambda \geq \lambda^{*}$ and $\gamma>\lambda-\lambda^{*}$, there exist $C, a_{4}$ and $\bar{L}$ such that, if $L \geq \bar{L}$ and $j \geq j_{L}$,

$$
\alpha_{j, L} \leq C e^{-a_{4}\left[\gamma-\left[\lambda-\lambda^{*}\right)\right] L^{d-1}}
$$

Proof. Note that

$$
\begin{aligned}
& P_{L}(N-j<M \leq N) \leq \\
& \leq P_{L}\left(y_{L} \geq \frac{\left(\lambda-\lambda^{*}\right) L^{d}+c_{L} L^{2}+x_{N} L^{d / 2}-j}{h_{L}}\right)
\end{aligned}
$$

Hence, if $j \leq j_{L}$, with $j_{L} / L^{d} \rightarrow \gamma$ as $L \rightarrow \infty$, and $\left|x_{N}\right| \leq L^{(d-1) / 2}$, so that $\left|x_{N}\right| L^{d / 2} / h_{L} \leq L^{-1 / 2} L^{d} / h_{L}$,

$$
P_{L}(N-j<M \leq N) \leq P_{L}\left(y_{L} \geq \frac{\left(\lambda-\lambda^{*}-\gamma-\delta_{L}\right) L^{d}}{h_{L}}\right)
$$


with $\delta_{L} \rightarrow 0$ as $L \rightarrow \infty$. On the other hand

$$
\frac{1}{L^{d / 2}} \sum_{N=0}^{\infty} e^{-\frac{\beta}{2} x_{N}^{2}} \mathbf{1}_{\left|x_{N}\right| \geq L^{(d-1) / 2}} \leq C e^{-\frac{\beta}{4} L^{d-1}}
$$

The bound (D.26) then easily follows from (D.8) and Lemma D.2 since the upper bound in (D.13) is independent of the $u_{2}$ value (equal to $+\infty$ in this case).

The bound (D.27) is proved in a similar way, by using the upper bound in (D.14) and the remark that $L^{d-1} \leq\left(L^{d} / h_{L}\right)^{2}$.

We have now all the technical ingredients to study the Laplace transform $F_{A, L}(\sigma)$ of the probability distribution of the random variables $y_{A, L}$ defined in (D.1). We have

$$
\begin{aligned}
F_{A, L}(\sigma) & =\frac{e^{\sigma \frac{L^{d} \rho_{A, L}}{h_{A, L}}}}{\Gamma_{\lambda, L}} \frac{1}{L^{d / 2}} \sum_{N \geq 0} e^{-\beta x_{N}^{2} / 2} \frac{1}{Q_{L}} \sum_{\left\{n_{j} \geq 0, j \geq 1\right\}} \mathbf{1}_{\sum j n_{j}=N} . \\
& \cdot \prod_{j \notin A} w\left(j, n_{j}\right) \prod_{j \in A} w\left(j, n_{j}\right) e^{-\sigma \frac{j n_{j}}{h_{A, L}}}
\end{aligned}
$$

By using (2.14) and the identity

$$
\begin{aligned}
& w(j, n) e^{-\sigma \frac{j n_{j}}{h_{A, L}}}= \\
& =\sum_{\left\{n^{\prime}, n^{\prime \prime} \geq 0\right\}} \mathbf{1}_{n^{\prime}+n^{\prime \prime}=n} \frac{1}{n^{\prime} !}\left[\frac{L^{d} a(\beta j, L) e^{-\sigma \frac{j}{h_{A, L}}}-1}{j}\right]^{n^{\prime}} \frac{1}{n^{\prime \prime} !}\left(\frac{1}{j}\right)^{n^{\prime \prime}}
\end{aligned}
$$

we see that

$$
F_{A, L}(\sigma)=G_{A, L}(\sigma) \frac{1}{\Gamma_{\lambda, L}} \frac{1}{L^{d / 2}} \sum_{N \geq 0} e^{-\beta x_{N}^{2} / 2} P_{A, L, \sigma}(M \leq N)
$$


where

$$
\begin{aligned}
P_{A, L, \sigma}(M) & =Q_{A, L, \sigma}^{-1} \sum_{\left\{n_{j} \geq 0, j \geq 1\right\}} \mathbf{1}_{\sum j n_{j}=M} \cdot \\
& \cdot \prod_{j \notin A} \tilde{w}\left(j, n_{j}\right) \prod_{j \in A} \tilde{w}\left(j, n_{j}, \sigma\right) \\
Q_{A, L, \sigma} & =\sum_{\left\{n_{j} \geq 0, j \geq 1\right\}} \prod_{j \notin A} \tilde{w}\left(j, n_{j}\right) \prod_{j \in A} \tilde{w}\left(j, n_{j}, \sigma\right) \\
\tilde{w}(j, n, \sigma) & =\frac{1}{n !}\left[\frac{L^{d} a(\beta j, L) e^{-\sigma \frac{j}{h_{A, L}}}-1}{j}\right]^{n} \\
G_{A, L}(\sigma) & =e^{\sigma^{\frac{L^{d} \rho_{A, L}}{h_{A, L}}} \frac{Q_{A, L, \sigma}}{Q_{L}}}
\end{aligned}
$$

Note that $P_{A, L, \sigma}(M)$ in general is not a probability distribution for any value of $\sigma$ (this is clear only for $\sigma$ real and negative). Moreover, in all the choices of the set $A$ we shall consider, $P_{A, L, \sigma}(M)$ is absolutely summable over $M$ and its sum is equal to 1 (which is formally true by definition). Hence, we can consider it as a finite complex measure on $\mathbb{R}$ (with support on a lattice set) and we shall study its convergence, as $L \rightarrow \infty$, to a measure with smooth density.

A few simple calculations show that

$$
\log Q_{A, L, \sigma}=\sum_{j \in A} \frac{L^{d} a(\beta j, L) e^{-\sigma \frac{j}{h_{A, L}}}-1}{j}+\sum_{j \notin A} \frac{L^{d} a(\beta j, L)-1}{j}
$$

so that

$$
\begin{gathered}
\log G_{A, L}(\sigma)=\sigma \frac{L^{d} \rho_{A, L}}{h_{A, L}}+\sum_{j \in A} \frac{L^{d} a(\beta j, L)}{j}\left(e^{-\sigma \frac{j}{h_{A, L}}}-1\right)= \\
=\sigma r_{A, L}^{*}+\sum_{j \in A} \frac{L^{d} a(\beta j, L)}{j}\left(e^{-\sigma \frac{j}{h_{A, L}}}-1+\sigma \frac{j}{h_{A, L}}\right)
\end{gathered}
$$


where

$$
r_{A, L}^{*}=\frac{L^{d}}{h_{A, L}} \sum_{j \in A} a(\beta j, L)\left(\alpha_{j, L}-1\right)
$$

It will also useful to consider the random variable

$$
y_{A, L, \sigma}=\frac{M-M_{A, L, \sigma}^{*}}{h_{L}}
$$

where $M$ is a random variable with measure $P_{A, L, \sigma}(M)$ and $h_{L}$ is defined as in (B.12).

The mean value of $M$ is given by

$$
\begin{aligned}
M_{A, L, \sigma}^{*} & \equiv \sum_{M=0}^{\infty} M P_{A, L, \sigma}(M)=\sum_{j \in A}\left(L^{d} a(\beta j, L) e^{-\sigma \frac{j}{h_{A, L}}}-1\right) \\
& +\sum_{j \notin A}\left(L^{d} a(\beta j, L)-1\right)
\end{aligned}
$$

By using (B.8) and (B.4), we see that

$$
M_{A, L, \sigma}^{*}=L^{d} \lambda_{L}^{*}+L^{d} \sum_{j \in A} a(\beta j, L)\left(e^{-\sigma_{\frac{j}{h_{A, L}}}}-1\right)
$$

so that

$$
\begin{gathered}
P_{A, L, \sigma}(M \leq N)= \\
P_{A, L, \sigma}\left(y_{A, L, \sigma} \leq \frac{L^{d}\left(\lambda-\lambda^{*}\right)+L^{d / 2} x_{N}+L^{2} c_{L}}{h_{L}}+y_{A, L, \sigma}^{*}\right) \\
y_{A, L, \sigma}^{*}=\frac{L^{d}}{h_{L}} \sum_{j \in A} a(\beta j, L)\left(1-e^{-\sigma \frac{j}{h_{A, L}}}\right)
\end{gathered}
$$

As in the proof of Theorem B.2, the limiting distribution of $y_{A, L, \sigma}$ will be obtained by studying the Laplace transform $H_{A, L, \sigma}(w)$ of its measure. We 
have

$$
\begin{aligned}
& \log H_{A, L, \sigma}(w)=\sum_{j=1}^{\infty} \frac{L^{d} a(\beta j, L)-1}{j}\left(e^{-w \frac{j}{h_{L}}}-1+w \frac{j}{h_{L}}\right)+ \\
&+\log R_{A, L, \sigma}(w) \\
& \log R_{A, L, \sigma}(w)=\sum_{j \in A} \frac{L^{d} a(\beta j, L)}{j}\left(e^{-\sigma \frac{j}{h_{A, L}}}-1\right)\left(e^{-w \frac{j}{h_{L}}}-1+w \frac{j}{h_{L}}\right)
\end{aligned}
$$

If we define $f_{A, L, \sigma}(t)=H_{A, L, \sigma}(-i t)$, we have also

$$
h_{L} P_{A, L, \sigma}(M)=\frac{1}{2 \pi} \int_{-\pi h_{L}}^{+\pi h_{L}} e^{-i t y_{A, L, \sigma}} f_{A, L, \sigma}(t)
$$

Moreover, if $\sigma=0$, the function $H_{A, L, \sigma}(w)$ has to coincide (as one could check by using the identity (A.4) and some easy algebra) with the function $F_{L}(w)$ defined in (B.18). It follows that

$$
\begin{aligned}
\log H_{A, L, \sigma}(w) & =\log F_{L}(w)+\log R_{A, L, \sigma}(w) \\
f_{A, L, \sigma}(t) & =f_{L}(t) R_{A, L, \sigma}(-i t)
\end{aligned}
$$

We shall consider some special cases for the set $A$. First of all, we consider the simplest one, that is the case where $A$ contains only one element; we prove the following Theorem.

Theorem D.4. If $A=\{j\}$ and $h_{A, L}=L^{d / 2}$, then, if $d \geq 3$ and $\lambda \geq \lambda^{*}$,

$$
\rho_{\{j\}, L} \underset{L \rightarrow \infty}{\longrightarrow} \rho_{j} \equiv(2 \pi \beta j)^{-d / 2}
$$

Moreover, if $d \geq 3$ and $\lambda>\lambda^{*}$ or $d=3,4$ and $\lambda=\lambda^{*}$, the probability distribution of $y_{A, L}$ converges, as $L \rightarrow \infty$, to a Gaussian distribution with variance $j \rho_{j}$. Finally, if $d \geq 5$ and $\lambda=\lambda^{*}$, the limiting distribution is still 
well defined, but its Laplace transform is given by

$$
\begin{aligned}
\lim _{L \rightarrow \infty} \log F_{\{j\}, L}(\sigma) & =-\sigma r^{*} j \rho_{j}+\frac{1}{2} \sigma^{2} j \rho_{j}+ \\
& +\log \left\{\frac{\int_{-\infty}^{+\infty} d x e^{-\beta x^{2} / 2} \int_{-\infty}^{x+\sigma j \rho_{j}} d y \rho(y)}{\int_{-\infty}^{+\infty} d x e^{-\beta x^{2} / 2} \int_{-\infty}^{x} d y \rho(y)}\right\}
\end{aligned}
$$

where

$$
r^{*}=\frac{\int_{-\infty}^{+\infty} d x e^{-\beta x^{2} / 2} \rho(x)}{\int_{-\infty}^{+\infty} d x e^{-\beta x^{2} / 2} \int_{-\infty}^{x} d y \rho(y)}
$$

Proof. By using the definition of $a(\beta j, L)$ in $(2.2)$, we see that, for any $j$,

$$
a(\beta j, L) \underset{L \rightarrow \infty}{\longrightarrow}(2 \pi \beta j)^{-d / 2}=\rho_{j}
$$

Moreover, by Lemma D.1 and (D.2), $\forall \lambda \geq \lambda^{*}$,

$$
\lim _{L \rightarrow \infty} \alpha_{j, L}=1 \Rightarrow \lim _{L \rightarrow \infty} \rho_{j, L}=\rho_{j}
$$

Let us now observe that, by (D.47), for any fixed $\sigma$ and $w$,

$$
\left|\log R_{\{j\}, L, \sigma}(w)\right| \leq C \frac{L^{d}}{L^{d / 2} h_{L}^{2}} j^{2-d / 2} \underset{L \rightarrow \infty}{\longrightarrow} 0
$$

Moreover, if we put $w=-i t, t \in \mathbb{R}$, for any fixed $\sigma$, we have

$$
\left|\log R_{\{j\}, L, \sigma}(-i t)\right| \leq C|t| \frac{L^{d}}{L^{d / 2} h_{L}} j^{1-d / 2} \leq C|t|
$$

Hence we see, by using (D.49) and the bound (B.30), that $\left|f_{A, L, \sigma}(t)\right| \leq$ $\exp \left(-C|t|^{3 / 2}\right)$. It is easy to prove that this bound is valid also for $f_{A, L, \sigma}^{\prime \prime}(t)$, which implies that the measure $P_{\{j\}, L, \sigma}(M)$ satisfies the analogous of (B.15) and (B.16) (we omit the details); and that $P_{\{j\}, L, \sigma}(y \leq \bar{y})$ converges to $P(y \leq \bar{y})$ for any $\bar{y}$, if $P(d y)$ is the limiting probability measure of Theorem B.2. On the other hand, we have, see (D.45),

$$
y_{\{j\}, L, \sigma}^{*} \underset{L \rightarrow \infty}{\longrightarrow}\left\{\begin{array}{lr}
0 & d=3,4 \\
\sigma j \rho_{j} & d \geq 5
\end{array}\right.
$$


Note also that, by Lemmas D.1 and D.3, see (D.40)

$$
r_{\{j\}, L}^{*} \underset{L \rightarrow \infty}{\longrightarrow}\left\{\begin{array}{lrll}
0 & d=3,4 & , & \lambda \geq \lambda^{*} \\
0 & d \geq 5, & \lambda>\lambda^{*} \\
-r^{*} j \rho_{j} & d \geq 5, & \lambda=\lambda^{*}
\end{array}\right.
$$

If $d=3$ or $d=4$, it follows, by using (D.33) and (D.39), that, for any $\lambda \geq \lambda^{*}$,

$$
\lim _{L \rightarrow \infty} \log F_{\{j\}, L}(\sigma)=\lim _{L \rightarrow \infty} \log G_{\{j\}, L}(\sigma)=\frac{1}{2} \sigma^{2} j \rho_{j}
$$

The same result is true, if $d \geq 5$ and $\lambda>\lambda^{*}$. However, if $d \geq 5$ and $\lambda=\lambda^{*}$, we get (D.51).

The fact that the Laplace transform converges for any $\sigma$ implies that also the characteristic function is convergent. As it is well known [3], this is sufficient to prove the convergence of the probability distribution of $y_{A, L}$.

We now consider a more interesting choice of the set $A$.

Theorem D.5. Let $A=\left\{1 \leq j \leq j_{L}\right\}$, with $j_{L}$ a monotone diverging function of $L$ such that $j_{L} \leq L^{2}$. Then, if $d \geq 3$ and $\lambda \geq \lambda^{*}$,

$$
\rho_{A, L} \underset{L \rightarrow \infty}{\longrightarrow} \sum_{j=1}^{\infty} \rho_{j}=\lambda^{*}
$$

Moreover, if $j_{L} / L^{2} \underset{L \rightarrow \infty}{\longrightarrow} 0$ and $\lambda>\lambda^{*}$, the probability distribution of $y_{A, L}$ converges, as $L \rightarrow \infty$, to a Gaussian distribution, provided we take

$$
h_{A, L}= \begin{cases}L^{2}\left(j_{L} / L^{2}\right)^{1 / 4} & d=3 \\ L^{2} \sqrt{\log j_{L}} & d=4 \\ L^{d / 2} & d \geq 5\end{cases}
$$

The same result is true, if $\lambda=\lambda^{*}$, provided $d=3$ or $d=4$ and $\lim _{L \rightarrow \infty}$ $\sqrt{\log j_{L} / \log L}=0$; in the other cases the limiting distribution is still well defined but it is not gaussian. 
Proof. By using (2.2), it is easy to prove that, if $j \leq L^{2}$, there is a constant $C$, independent of $j$ and $L$, such that

$$
a(\beta j, L) \leq C \rho_{j} \quad, \quad 0<a(\beta j, L)-\rho_{j} \leq C \rho_{j} e^{-L^{2} /(2 \beta j)}
$$

Hence, since $0 \leq \alpha_{j, L} \leq 1$,

$$
0 \leq \sum_{j \leq j_{L}}\left(\rho_{j, L}-\rho_{j} \alpha_{j, L}\right) \leq C \sum_{j \leq L^{2}} j^{-d / 2} e^{-L^{2} /(2 \beta j)} \leq C L^{2-d} \underset{L \rightarrow \infty}{\longrightarrow} 0
$$

On the other hand, by (D.6),

$$
0 \leq \sum_{j \leq j_{L}} \rho_{j}\left(1-\alpha_{j, L}\right) \leq \frac{C}{h_{L}} \sum_{j \leq L^{2}} j^{-d / 2+1} \underset{L \rightarrow \infty}{\longrightarrow} 0
$$

(D.60) is an easy consequence of the last two bounds.

In order to study the limiting distribution of $y_{A, L}$, we proceed as in the proof of Theorem D.4. First of all we observe that, by (D.47), if $h_{A, L}$ is chosen as in (D.61), for any fixed $\sigma$ and $w$,

$$
\left|\log R_{A, L, \sigma}(w)\right| \leq C \frac{L^{d}}{h_{A, L} h_{L}^{2}} \sum_{j=1}^{j_{L}} j^{2-d / 2} \underset{L \rightarrow \infty}{\longrightarrow} 0
$$

Moreover, if we put $w=-i t, t \in \mathbb{R}$, for any fixed $\sigma$, we have

$$
\left|\log R_{\{j\}, L, \sigma}(-i t)\right| \leq C|t| \frac{L^{d}}{h_{A, L} h_{L}} \sum_{j=1}^{j_{L}} j^{1-d / 2} \leq C|t|
$$

Once again, by proceeding as in the proof of Theorem D.4, one can prove that the measure $P_{A, L, \sigma}(M)$ satisfies the analogous of (B.15) and (B.16); we omit the details. This implies the convergence of $P_{A, L, \sigma}(y \leq \bar{y})$ to $P(y \leq \bar{y})$ for any $\bar{y}$, if $P(d y)$ is the limiting probability measure of Theorem B.2. On the other hand, we have, see (D.45),

$$
y_{A, L, \sigma}^{*} \underset{L \rightarrow \infty}{\longrightarrow} \begin{cases}0 & d=3 \\ \sigma\left(2 \pi^{2} \beta\right)^{-2} \lim _{L \rightarrow \infty} \sqrt{\log j_{L} / \log L} & d=4 \\ \sigma \sum_{j=1}^{\infty} j \rho_{j} & d \geq 5\end{cases}
$$


Let us now suppose that $\lambda>\lambda^{*}$. Then, by using (D.26), we can easily show that, for any $d \geq 3, r_{A, L}^{*}$, (see (D.40)) goes to 0 as $L \rightarrow \infty$. It is also very easy to show that the factor which multiplies $G_{A, L}(\sigma)$ in the r.h.s. of (D.33) goes to 1 as $L \rightarrow \infty$. Hence, by using (D.39), we get

$$
\begin{aligned}
& \lim _{L \rightarrow \infty} \log F_{A, L}(\sigma)=\lim _{L \rightarrow \infty} \log G_{A, L}(\sigma)= \\
& \lim _{L \rightarrow \infty} \sum_{j=1}^{j_{L}} \frac{L^{d} a(\beta j, L)}{j}\left(e^{-\sigma \frac{j}{h_{A, L}}}-1+\sigma \frac{j}{h_{A, L}}\right)
\end{aligned}
$$

If we insert the value of $h_{A, L}$ given in (D.61) in the r.h.s. of this equation, we can see that only the terms of order $\sigma^{2}$ survive in the limit $L \rightarrow \infty$. Moreover, the fact that the Laplace transform converges for any $\sigma$ implies that also the characteristic function is convergent. Therefore the limiting distribution of $y_{A, L}$ is well defined and is gaussian; we can also easily calculate its variance.

If $\lambda=\lambda^{*}$, we get a different result for $d \geq 4$, because we can see, by using Lemma D.1, that

$$
r_{A, L}^{*} \underset{L \rightarrow \infty}{\longrightarrow} \begin{cases}0 & d=3 \\ -2 \rho_{0}\left(2 \pi^{2} \beta\right)^{-2} \lim _{L \rightarrow \infty} \sqrt{\log j_{L} / \log L} & d=4 \\ -r^{*} \sum_{j=1}^{\infty} j \rho_{j} & d \geq 5\end{cases}
$$

Moreover, the factor which multiplies $G_{A, L}(\sigma)$ in the r.h.s. of (D.33) is still convergent, but it goes to 1 as $L \rightarrow \infty$ only for $d=3$ and for $d=4$, if $\lim _{L \rightarrow \infty} \sqrt{\log j_{L} / \log L}=0$. It follows that the limiting distribution of $y_{A, L}$ is still well defined and its Laplace transform can be explicitly calculated; however, it is gaussian only for $d=3$ and for $d=4$, if $\lim _{L \rightarrow \infty} \sqrt{\log j_{L} / \log L}=0$. 


\section{Appendix E. Large deviations for "short loops"}

In this appendix we consider $d \geq 3$ and $\lambda>\lambda^{*}$; our main result is stated in Theorem E.3

Given any finite subset $A$ of $\mathbb{N}^{+}$, we define

$$
S_{A, L}(v, \delta) \equiv P_{\lambda, L}^{m f}\left[L^{d}\left(\rho_{A, L}+v-\delta\right) \leq \sum_{j \in A} j n_{j} \leq L^{d}\left(\rho_{A, L}+v+\delta\right)\right]
$$

with $\rho_{A, L}$ defined as in (D.1). We want to show that, if $A=\{j\}$ or $A=$ $\left\{j \leq j_{L}\right\}$, with $j_{L}$ a monotone diverging function of $L$, such that $j_{L} \leq L^{2}$, we can evaluate the behavior for $L \rightarrow \infty$ of the r.h.s. of (E.1) by substituting the measure $P_{\lambda, L}^{m f}$ with the measure, independent of $\lambda$ (recall that $\lambda>\lambda^{*}$ in this section)

$$
P_{A, L}\left(n_{j}, j \in A\right)=Z_{A, L}^{-1} \prod_{j \in A} w\left(j, n_{j}\right)
$$

We shall consider in detail only the case $A=\left\{j \leq j_{L}\right\}$; the other case can be treated in a similar (simpler) way.

Let us define $\Pi_{A, L}(t)=\log F_{A, L}\left(t h_{A, L}\right)$ and

$$
\begin{aligned}
\tilde{\Pi}_{A, L}(t) & =\log G_{A, L}\left(t h_{A, L}\right)-t h_{A, L} r_{A, L}^{*}= \\
& =\sum_{j \in A} \frac{L^{d} a(\beta j, L)}{j}\left(e^{-t j}-1+t j\right)
\end{aligned}
$$

where $F_{A, L}(\sigma)$ and $G_{A, L}(\sigma)$ are defined as in (D.33) and $r_{A, L}^{*}$ is defined as in (D.40). Choose $t \equiv t_{L}(v)$ so that

$$
-\tilde{\Pi}_{A, L}^{\prime}(t)=v L^{d}
$$

Lemma E.1. Let $A=\left\{1 \leq j \leq j_{L}\right\}$, with $j_{L}$ a monotone diverging function of $L$ such that $j_{L} \leq L^{2}$. Then the equation (E.4) has a solution for any $v>-\lambda^{*}$. Moreover, if $0>v>-\lambda^{*}, t_{L}(v)$ has a finite positive limit 
$t(v)$ for $L \rightarrow \infty$, such that, for $v \rightarrow 0$ and suitable constants $c_{d}$

$$
t(v) \sim \begin{cases}c_{3} v^{2} & d=3 \\ c_{4} v / \log (1 / v) & d=4 \\ c_{d} v & d \geq 5\end{cases}
$$

while, if $v>0, t_{L}(v)<0$ and

$$
\lim _{L \rightarrow \infty} \frac{j_{L}\left|t_{L}(v)\right|}{\log \left(v j_{L}^{d / 2-1}\right)}=1
$$

Proof. By using (D.39), we see that equation (E.4) can be written as

$$
H_{L}(t) \equiv \sum_{j=1}^{j_{L}} a(\beta j, L)\left(e^{-t j}-1\right)=v
$$

Note that the sign of $t$ is the opposite of the sign of $v$. Let us consider first the case $v<0$. In this case $t>0$ and $H_{L}(t) \rightarrow-\sum_{j \leq j_{L}} a(\beta j, L)$, as $t \rightarrow+\infty$; since $\lim _{L \rightarrow \infty} \sum_{j \leq j_{L}} a(\beta j, L)=\lambda^{*}$, equation (E.7) has a unique solution $t_{L}(v)$ only if $v>-\lambda^{*}$. It is an easy exercise to show that $t_{L}(v)$ converges, as $L \rightarrow \infty$, to a limit $t(v)$, verifying (E.5).

If $v>0$, there is a unique negative solution $t_{L}(v)$ of (E.7) for any $v$, since $H_{L}(t) \rightarrow+\infty$ as $t \rightarrow-\infty$, and it is easy to see that $\lim _{L \rightarrow \infty} t_{L}(v)=0$. A more careful analysis shows that equation (E.6) is verified.

Lemma E.2. Let $A=\left\{1 \leq j \leq j_{L}\right\}$, with $j_{L}$ a monotone diverging function of $L$ such that $j_{L} \leq L^{2}$. Then, if $t$ is defined as in (E.4), there exists $\eta>0$ such that, for $L$ large enough,

$$
\begin{aligned}
F_{A, L}\left(t h_{A, L}\right) & =G_{A, L}\left(t h_{A, L}\right)\left[1+\delta_{L}(t)\right], \quad\left|\delta_{L}(t)\right| \leq e^{-L^{\eta}} \\
\Pi_{A, L}^{\prime}(t) & =\tilde{\Pi}_{A, L}^{\prime}(t)+\delta_{L}(t) \quad, \quad\left|\delta_{L}(t)\right| \leq e^{-L^{\eta}}
\end{aligned}
$$


Proof. By using (D.33), we see that (E.8) is satisfied, if we define

$$
\begin{aligned}
\delta_{L}(t) & =\delta_{L, 1}+\delta_{L, 2}(t) \\
\delta_{L, 1} & =\frac{1}{\Gamma_{\lambda, L}} \frac{1}{L^{d / 2}} \sum_{N \geq 0} e^{-\beta x_{N}^{2} / 2}-1 \\
\delta_{L, 2}(t) & =-\frac{1}{\Gamma_{\lambda, L}} \frac{1}{L^{d / 2}} \sum_{N \geq 0} e^{-\beta x_{N}^{2} / 2} P_{A, L, t}(M>N)
\end{aligned}
$$

where $P_{A, L, t}(M)$ is the measure defined in (D.34), with $\sigma=t h_{A, L}, t$ being the solution of (E.7). By using (C.3), we see that

$$
\delta_{L, 1}=\frac{1}{\Gamma_{\lambda, L}} \frac{1}{L^{d / 2}} \sum_{N \geq 0} e^{-\beta x_{N}^{2} / 2} P_{L}(M>N)
$$

where $P_{L}(M)$ is the probability measure defined in (B.3). By using (C.4) and the large deviation estimates of Lemma D.2, it is easy to prove that $\left|\delta_{L, 1}\right| \leq \exp \left(-L^{\eta}\right)$, with $0<\eta<d-2$.

In order to prove a similar bound for $\delta_{L, 2}(t)$, we need a large deviation estimate for the measure $P_{A, L, t}(M)$. Let us consider first the case $v>0$, that is $t<0$, when $P_{A, L, t}(M)$ is a probability measure. In this case, if $\lambda-\lambda^{*}-v>0$ and $\left|x_{N}\right| \leq L^{d / 4}$, by using (D.43) and (E.7), we see that, for $L$ large enough

$$
\begin{aligned}
& P_{A, L, t}(M>N)=P_{A, L, t}\left(M-M_{A, L, t}^{*}>L^{d}\left(\lambda-\lambda^{*}-v\right)+\right. \\
& \left.+L^{d / 2} x_{N}+L^{2} c_{L}+h_{A, L} r_{A, L}^{*}\right) \leq P_{A, L, t}\left(M-M_{A, L, t}^{*}>L^{d} \epsilon\right)
\end{aligned}
$$

if $0<\epsilon<\lambda-\lambda^{*}-v$, so that, for $L$ large enough,

$$
\left|\delta_{L, 2}(t)\right| \leq 2 e^{-\beta L^{d / 2} / 2}+2 P_{A, L, t}\left(M-M_{A, L, t}^{*}>L^{d} \epsilon\right)
$$

Let us consider the Laplace transform $H_{L, t}(w)$ of the random variable $y=M-M_{A, L, t}^{*} ;$ it is related to the analogous function $H_{A, L, \sigma}(w)$ defined in (D.46) by the relation

$$
H_{L, t}(w)=H_{A, L, t h_{A, L}}\left(w h_{L}\right)
$$


By proceeding as in the proof of Lemma D.2, we can write

$$
P_{A, L, t}\left(M-M_{A, L, t}^{*}>L^{d} \epsilon\right)=e^{\Pi_{L, t}(w)} \int_{\epsilon L^{d}}^{+\infty} e^{w y} P_{L, t, w}(d y)
$$

where $\Pi_{L, t}(w)=\log H_{L, t}(w)$ and $P_{L, t, w}(d y)=e^{-w y} P_{L, t}(d y) / H_{L, t}(w)$. We now fix $w$ so that $-\Pi_{L, t}^{\prime}(w)=\epsilon L^{d}$; it is to see that this condition can be written as

$$
\begin{aligned}
\epsilon L^{d} & =L^{d} \sum_{j=1}^{j_{L}} a(\beta j, L)\left(e^{-t j}-1\right)\left(1-e^{-w j}\right)+ \\
& +\sum_{j=1}^{\infty}\left(L^{d} a(\beta j, L)-1\right)\left(1-e^{-w j}\right)
\end{aligned}
$$

Since $t<0$, the solution of this equation has to be negative and one can show that $\Pi_{L, t}(w)+w \epsilon L^{d}=\Pi_{L, t}(w)-w \Pi_{L, t}^{\prime}(w)$ is negative and of order $L^{d-2}$. It follows that

$$
P_{A, L, t}\left(M-M_{A, L, t}^{*}>L^{d} \epsilon\right) \leq e^{\Pi_{L, t}(w)-w \Pi_{L, t}^{\prime}(w)} \leq e^{-c L^{d-2}}
$$

for some $c>0$.

The case $v<0$, that is $t>0$, is a bit more involved, since in this case $P_{A, L, t}(M)$ is not necessarily a probability measure. However, it is easy to prove, by using (D.34), that

$$
\sum_{M=0}^{\infty}\left|P_{A, L, t}(M)\right| \leq e^{2 \log j_{L}}
$$

so that, instead of the bound (E.15), we have

$$
\left|\delta_{L, 2}(t)\right| \leq 2 e^{-\beta L^{d / 2} / 2+2 \log j_{l}}+2\left|P_{A, L, t}\left(M-M_{A, L, t}^{*}>L^{d} \epsilon\right)\right|
$$

Moreover, since it is still true that $\Pi_{L, t}(w)-w \Pi_{L, t}^{\prime}(w)$ is negative and of order $L^{d-2}$, instead of bound (E.19), we get

$$
\left|P_{A, L, t}\left(M-M_{A, L, t}^{*}>L^{d} \epsilon\right)\right| \leq e^{-c L^{d-2}} \sum_{M=0}^{\infty}\left|P_{L, t, w}(M)\right|
$$


which is a negligible difference, since $|w| L^{2} \leq c$, so that, as one can easily check,

$$
\sum_{M=0}^{\infty}\left|P_{L, t, w}(M)\right| \leq e^{2 e^{c j_{L} / L^{2}} \log j_{L}}
$$

We still have to prove (E.9). By putting $\sigma=t h_{A, L}$ in (D.33) and by doing the derivative with respect to $t$, we get

$$
\begin{aligned}
& \frac{d}{d t} F_{A, L}\left(t h_{A, L}\right)=L^{d} \rho_{A, L} F_{A, L}\left(t h_{A, L}\right)- \\
& -G_{A, L}\left(t h_{A, L}\right) \sum_{j=1}^{j_{L}} L^{d} a(\beta j, L) e^{-t j}\left[1+\delta_{L, 2, k}(t)+\delta_{L, 1}\right]
\end{aligned}
$$

where $\delta_{L, 1}$ is defined as in (E.11) and

$$
\delta_{L, 2, k}(t)=-\frac{1}{\Gamma_{\lambda, L}} \frac{1}{L^{d / 2}} \sum_{N \geq 0} e^{-\beta x_{N}^{2} / 2} P_{A, L, t}(M>N-k)
$$

Since $k \leq j_{L} \leq L^{2}$, we can find as before that $\left|\delta_{L, 2, k}(t)\right| \leq e^{-L^{\eta}}$. On the other hand $\sum_{j \leq j_{L}} L^{d} a(\beta j, L) e^{-t j}$ is always of order $L^{d}$. It follows that

$$
\begin{aligned}
\Pi_{A, L}^{\prime}(t) & =\frac{\frac{d}{d t} F_{A, L}\left(t h_{A, L}\right)}{F_{A, L}\left(t h_{A, L}\right)}=L^{d} \rho_{A, L}-\sum_{j=1}^{j_{L}} L^{d} a(\beta j, L) e^{-t j}+O\left(e^{-L^{\eta}}\right) \\
& =\tilde{\Pi}_{A, L}^{\prime}(t)+O\left(e^{-L^{\eta}}\right)
\end{aligned}
$$

Theorem E.3. If $\mathcal{A}_{L, \delta}^{(4)}(v)$ is defined as in (5.7) and $0<v<\lambda-\lambda^{*}$, then

$$
v-\delta \leq-\lim _{L \rightarrow \infty} \frac{1}{L^{d}} \frac{j_{L}}{\log \left(v j_{L}^{d / 2-1}\right)} \log P_{\lambda, L}^{m f}\left(\mathcal{A}_{L, \delta}^{(4)}\right) \leq u+\delta
$$

while, if $-\lambda^{*}<v<0$, there are positive functions $t_{d}(v)$ and $a_{d}(v)$, depending on $d$, such that

$$
a_{d}(v)-t_{d}(v) \delta \leq-\lim _{L \rightarrow \infty} \frac{1}{L^{d}} \log P_{\lambda, L}^{m f}\left(\mathcal{A}_{L, \delta}^{(4)}\right) \leq a_{d}(v)+t_{d}(v) \delta
$$


Moreover, the two bounds above do not change if we substitute $P_{\lambda, L}^{m f}\left(\mathcal{A}_{L, \delta}^{(4)}\right)$ with $\tilde{\Pi}_{A, L}\left(t_{L}(v)\right)-t \tilde{\Pi}_{A, L}^{\prime}\left(t_{L}(v)\right), t_{L}(v)$ being the solution of (E.4).

Proof. By (E.1) $P_{\lambda, L}^{m f}\left(\mathcal{A}_{L, \delta}^{(4)}\right)=S_{A, L}(v, \delta)$. By proceeding as in the proof of Lemma D.2, we can write

$$
S_{A, L}(v, \delta)=e^{\Pi_{A, L}(t)-t \Pi_{A, L}^{\prime}(t)} \int_{(v-\delta) L^{d}}^{(v+\delta) L^{d}} e^{t\left(y-v L^{d}\right)} P_{A, L, t}(d y)
$$

where $t$ is chosen so that $v L^{d}=-\Pi_{A, L}^{\prime}(t)$. By using Lemma E.2, we see that $\Pi_{A, L}(t)$ and its derivative can be substituted with $\tilde{\Pi}_{A, L}(t)$ and $\tilde{\Pi}_{A, L}^{\prime}(t)$, and that $t$ can be taken as the solution of (E.4), without changing the asymptotic behavior of $S_{A, L}(v, \delta)$,

Let us consider first the case $0<v<\lambda-\lambda^{*}$. It is easy to check that, in this case, $\tilde{\Pi}_{A, L}(t)$ is negligible, for $L \rightarrow \infty$, with respect to $|t| v L^{d}$, so that we get immediately, by using also (E.6), the lower bound in (E.27). In order to prove the upper bound, we need also a lower bound on $\int_{(v-\delta) L^{d}}^{(v+\delta) P_{A, L, t}^{d}}(d y)$, which can be obtained by studying the Laplace transform of $P_{A, L, t}(d y)$; this analysis shows that $\int_{(v-\delta) L^{d}}^{(v+\delta) L^{d}} P_{A, L, t}(d y) \rightarrow 1$ as $L \rightarrow \infty$.

If $-\lambda^{*}<v<0$, as shown in Lemma E.1, the solution of (E.4) converges, as $L \rightarrow \infty$, to the function $t(v)$ defined in (E.5). Moreover, it is easy to check that, up to negligible corrections, for any fixed $t$,

$$
\lim _{L \rightarrow \infty} L^{-d}\left[\Pi_{A, L}(t)-t \Pi_{A, L}^{\prime}(t)\right]=-a_{d}(v)
$$

where $a_{d}(v)$ is a positive function equal to

$$
a_{d}(v)=\sum_{j=1}^{\infty} \frac{1}{(2 \pi \beta j)^{d / 2}}\left(\frac{1-e^{-t j}}{j}-t e^{-t j}\right)
$$

Since it is still true that $\int_{(v-\delta) L^{d}}^{\left(v+\delta L^{d}\right.} P_{A, L, t}(d y) \rightarrow 1$ as $L \rightarrow \infty$, we get the bound (E.28). 


\section{Appendix F. Proof of Theorem 4.1}

By (D.2):

$$
\begin{aligned}
E_{\lambda, L}^{m f}\left(y_{\delta, L}\right) & =\frac{1}{L^{d}} \sum_{j \geq \delta L^{d}} L^{d} a(\beta j, L) \alpha_{j, L} \\
E_{\lambda, L}^{m f}\left(X_{\delta, L}\right) & =\sum_{j \geq \delta L^{d}} \frac{L^{d} a(\beta j, L)}{j} \alpha_{j, L} \\
E_{\lambda, L}^{m f}\left(X_{L}\right) & =\sum_{j \geq L^{2}} \frac{L^{d} a(\beta j, L)}{j} \alpha_{j, L}
\end{aligned}
$$

On the other hand, by (2.3),

$$
\left|L^{d} a(\beta j, L)-1\right| \leq C \exp \left(-C j / L^{2}\right) \quad, \quad \forall j \geq L^{2}
$$

which allows us to prove very easily the limits in (4.5) and (4.7), by using Lemma D.3.

The arguments given at the beginning of section $\mathrm{D}$ can be used to prove also that

$$
\begin{gathered}
E_{\lambda, L}^{m f}\left(n_{j_{1}} n_{j_{2}}\right)=\frac{L^{d} a\left(\beta j_{1}, L\right)}{j_{1}} \frac{L^{d} a\left(\beta j_{2}, L\right)}{j_{2}} \alpha_{j_{1}+j_{2}, L} \quad, \quad \text { if } j_{1} \neq j_{2} \\
E_{\lambda, L}^{m f}\left(n_{j}^{2}\right)=E_{\lambda, L}^{m f}\left(n_{j}\left(n_{j}-1\right)\right)+E_{\lambda, L}^{m f}\left(n_{j}\right)= \\
=\left(\frac{L^{d} a(\beta j, L)}{j}\right)^{2} \alpha_{2 j, L}+\frac{L^{d} a(\beta j, L)}{j} \alpha_{j, L}
\end{gathered}
$$

It follows that

$$
\begin{aligned}
& E_{\lambda, L}^{m f}\left(y_{\delta, L}^{2}\right)-E_{\lambda, L}^{m f}\left(y_{\delta, L}\right)^{2}= \\
& \sum_{j_{1}, j_{2} \geq \delta L^{d}} a\left(\beta j_{1}, L\right) a\left(\beta j_{2}, L\right)\left(\alpha_{j_{1}+j_{2}, L}-\alpha_{j_{1}, L} \alpha_{j_{2}, L}\right)+\sum_{j \geq \delta L^{d}} j a(\beta j, L) \alpha_{j, L}
\end{aligned}
$$


The limit (4.6) follows through some simple calculations from this identity, the bound (F.2) and Lemma D.3. In a similar way, one can prove also (4.8), (4.9) and (4.10).

We still have to prove (4.11). The previous arguments imply that, if $\xi \in(1 / 2,1]$ and $j>\xi\left(\lambda-\lambda^{*}\right) L^{d}$, then

$$
\lim _{L \rightarrow \infty} P_{\lambda, L}^{m f}\left(n_{j}=1\right)=\lim _{L \rightarrow \infty} E_{\lambda, L}^{m f}\left(n_{j}\right)
$$

and

$$
\lim _{L \rightarrow \infty} P_{\lambda, L}^{m f}\left(X_{\xi\left(\lambda-\lambda^{*}\right)} \geq 1\right)=\lim _{L \rightarrow \infty} \sum_{j>\xi\left(\lambda-\lambda^{*}\right) L^{d}} P_{\lambda, L}^{m f}\left(n_{j}=1\right)=-\log \xi
$$

\section{Appendix G. The limit functional}

Theorem G.1. There is a non decreasing, negative, continuous function $\lambda_{0}(\lambda)$ on $\mathbb{R}$, strictly negative in $d \leq 2$, such that

$$
\begin{gathered}
\pi(\lambda):=-\inf _{\underline{\rho}} F_{\lambda}(\underline{\rho})=\frac{\left[\lambda-\lambda_{0}(\lambda)\right]^{2}}{2}+\pi_{\lambda_{0}(\lambda)}^{0} \\
\pi_{\lambda_{0}(\lambda)}^{0}=\beta^{-1} \sum_{j} \frac{\rho_{j}^{*}}{j} e^{\lambda_{0}(\lambda) \beta j}
\end{gathered}
$$

Moreover, $\rho(\lambda):=\lambda-\lambda_{0}(\lambda)$, is a positive, strictly increasing, continuous function of $\lambda$ with range the whole $(0, \infty)$ and

$$
\inf _{\underline{\rho}} F_{\lambda}(\underline{\rho})=\inf _{\underline{\rho}: \sum_{j} \rho_{j}=\rho(\lambda)} F_{\lambda}(\underline{\rho})
$$


Remark 1. In $[\mathbf{1 0}]$ it is proved that the gran canonical mean field, thermodynamical pressure coincides with $\pi(\lambda)$, namely

$$
\pi(\lambda)=\lim _{L \rightarrow \infty} \frac{1}{L^{d}} \Xi_{\lambda, L}^{m f}
$$

thus proving our claim in the text that the computation of the pressure using the limit functional gives the correct result.

Remark 2. The proof of Theorem G.1 identifies the function $\lambda_{0}(\lambda)$ as follows. There exists a dimension dependent constant $\lambda^{*}$, equal to $+\infty$ in $d \leq 2$ and $<\infty$ in $d \geq 3$ such that for $\lambda \leq \lambda^{*}$ (by which we will mean, here and in the sequel, any $\lambda$ in $d \leq 2$ and $\lambda \leq \lambda^{*}$ in $d \geq 3$ ) there is a unique solution $\lambda_{0}$ of

$$
\lambda-\lambda_{0}=\sum_{j} \rho_{j}^{*} e^{\lambda_{0} \beta j}
$$

This solution, which depends on $\lambda$, coincides with the function $\lambda_{0}(\lambda)$ of Theorem G.1 for $\lambda \leq \lambda^{*}$, while the latter is identically 0 for $\lambda>\lambda^{*}$.

We will also prove that the inf in (G.1) is a minimum when $\lambda \leq \lambda^{*}$, and, in such a case, the minimizer is unique and given by

$$
\rho_{j}=\rho_{j}^{*} e^{\lambda_{0} \beta j}
$$

in agreement with (G.3), because $\sum_{j} \rho_{j}^{*} e^{\lambda_{0} \beta j}=\rho(\lambda)$ by (G.5).

The r.h.s. of (G.6) is the equilibrium density of $j$ loops in the free, gran canonical ensemble with chemical potential $\lambda_{0}$, so that $\lambda_{0}$ has the meaning of an effective chemical potential.

If $d \geq 3$ and $\lambda>\lambda^{*}$, the inf in (G.1) is not attained and there is a finite fraction of the density which "concentrates on infinitely long loops".

By (G.3), $\rho(\lambda)$ can be interpreted as the equilibrium density when the chemical potential is $\lambda$; since the range of $\rho(\lambda)$ is the whole $(0, \infty)$, there is no "forbidden interval" for the equilibrium density, namely there is no first 
order phase transition in our system. This is due to the assumption that the interaction energy $u^{2} / 2, u=\sum_{j} \rho_{j}$, is convex.
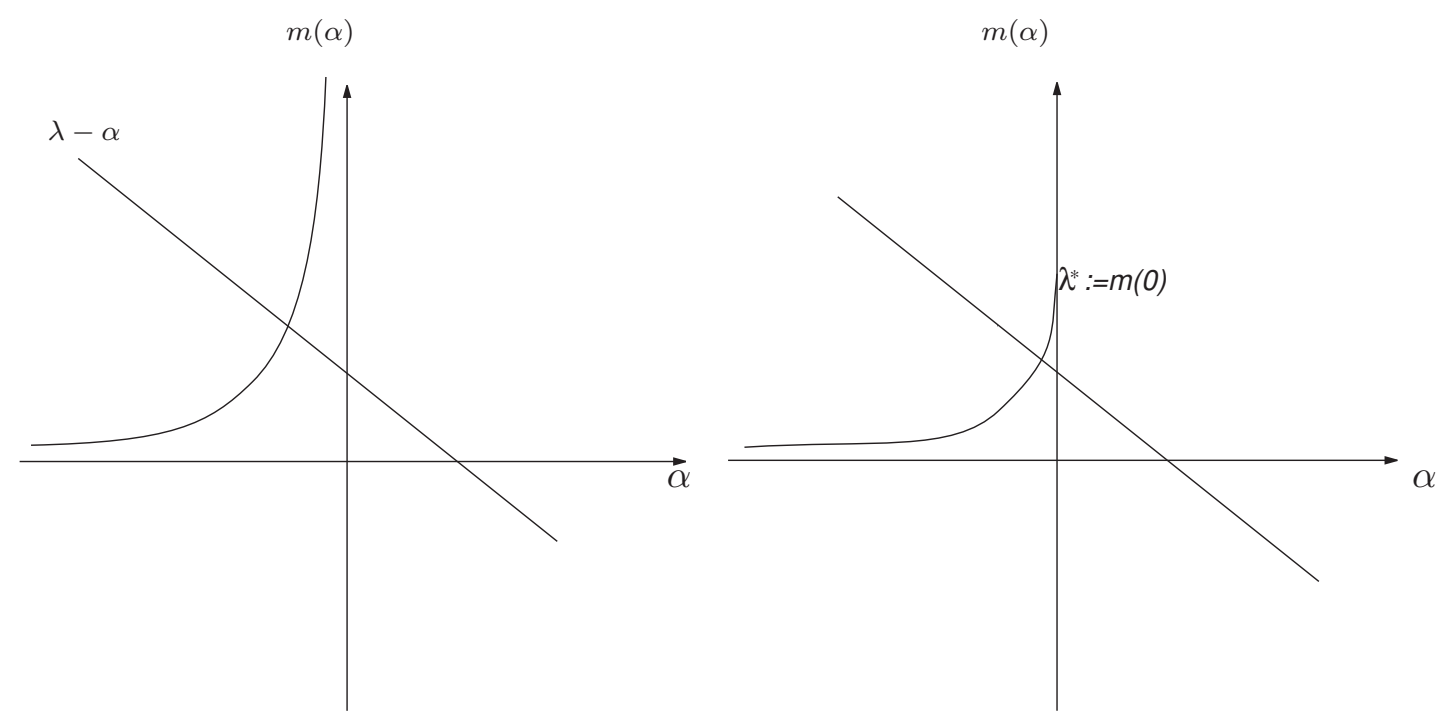

Figure 1. The two graphs, on the left and right, refer respectively to $d \leq 2$ and $d \geq 3$.

Proof of Theorem G.1 - Call $m(\alpha)=\sum_{j} \rho_{j}^{*} e^{\beta \alpha j}, \alpha<0$ in $d \leq 2$ and $\alpha \leq 0$ in $d \geq 3$. The graph of $m(\alpha)$ is as in Fig. 1 , having put $\lambda^{*}=m(0)$ in $d \geq 3$. Existence and uniqueness of the solution $\lambda_{0}$ of (G.5) for $\lambda \leq \lambda^{*}$ follow from monotonicity of $m(\cdot)$. Graphically $\lambda_{0}$ is the $\alpha$-coordinate of the intersection point in Fig. 1

Let us next prove (G.1)-(G.2) when $\lambda \leq \lambda^{*}$. With $\lambda_{0}$ as in (G.5) we write

$$
F_{\lambda}(\underline{\rho})=\left[\frac{\rho^{2}}{2}-\left(\lambda-\lambda_{0}\right) \rho\right]+\left[-\lambda_{0} \rho-\beta^{-1} S(\underline{\rho})\right], \quad \rho=\sum_{j} \rho_{j}
$$

Then

$$
F_{\lambda}(\underline{\rho}) \geq \inf _{\underline{\rho}}\left\{\frac{\rho^{2}}{2}-\left(\lambda-\lambda_{0}\right) \rho\right\}+\inf _{\underline{\rho}}\left\{-\lambda_{0} \rho-\beta^{-1} S(\underline{\rho})\right\}
$$


We have

$$
\inf _{\underline{\rho}}\left\{-\lambda_{0} \rho-\beta^{-1} S(\underline{\rho})\right\}=-\pi_{\lambda_{0}}^{0}
$$

with a unique minimizer given by (G.6). On the other hand

$$
\inf _{\underline{\rho}}\left\{\frac{\rho^{2}}{2}-\left(\lambda-\lambda_{0}\right) \rho\right\}=-\frac{\left(\lambda-\lambda_{0}\right)^{2}}{2}
$$

with minimizer any $\underline{\rho}$ such that $\sum_{j} \rho_{j}=\lambda-\lambda_{0}$. By (G.5), the previous minimizer $\underline{\rho}$, given by (G.6), satisfies such a condition, hence (G.1) and (G.3).

Suppose next that $d \geq 3$ and $\lambda>\lambda^{*}$. Then

$$
F_{\lambda}(\underline{\rho}) \geq \inf _{\underline{\rho}}\left\{\frac{\rho^{2}}{2}-\lambda \rho\right\}+\inf _{\underline{\rho}}\left\{-\beta^{-1} S(\underline{\rho})\right\} \geq-\frac{\lambda^{2}}{2}-\pi_{0}^{0}
$$

where the minimizers of the first inf are those $\underline{\rho}$ such that $\sum_{j} \rho_{j}=\lambda$, while the minimizer of the second inf is unique and given by $\left\{\rho_{j}^{*}\right\}$. We thus need to show that there is a sequence $\underline{\rho}^{(n)}$ such that $\sum_{j} \rho_{j}^{(n)}=\lambda$ and

$$
\lim _{n \rightarrow \infty} S\left(\underline{\rho}^{(n)}\right)=S\left(\underline{\rho}^{*}\right)
$$

Let $a:=\lambda-\rho^{*}$ be the excess mass, $\lambda$ being the mass $\rho$ which minimizes $\rho^{2} / 2-\lambda \rho$ and $\rho^{*}=\sum_{j} \rho_{j}^{*}$. Set

$$
\underline{\rho}_{j}^{(n)}= \begin{cases}\rho_{j}^{*} & \text { if } j \neq n \\ \rho_{j}^{*}+a & \text { if } j=n\end{cases}
$$

Then

$$
S\left(\underline{\rho}^{(n)}\right)=S\left(\underline{\rho}^{*}\right)+\left(\frac{\rho_{n}^{*}}{n}+\left(\frac{\rho_{n}^{*}}{n}+\frac{a}{n}\right)\left(\log \left(\frac{\rho_{n}^{*}+a}{\rho_{n}^{*}}\right)-1\right)\right)
$$

with the last bracket vanishing as $n \rightarrow \infty$. 
(G.1), (G.2) and (G.3) are therefore proved. We will next show that $\rho(\lambda)$ is continuous, strictly increasing, and with range $(0, \infty)$. Let $\lambda<\lambda^{*}$ and $\lambda_{0}^{\prime}$ the derivative of $\lambda_{0}$ w.r.t. $\lambda$. Then

$$
\frac{d \rho(\lambda)}{d \lambda}=1-\lambda_{0}^{\prime}
$$

and, by differentiating (G.5),

$$
\lambda_{0}^{\prime}\left(\beta \sum_{j} j \rho_{j}^{*} e^{\lambda_{0} \beta j}+1\right)=1
$$

Hence $\lambda_{0}(\cdot)$ is a non decreasing function, as claimed in Theorem G.1; moreover, since $\lambda_{0}^{\prime}<1, \frac{d \rho(\lambda)}{d \lambda}>0$, for $\lambda<\lambda^{*}$. For $\lambda>\lambda^{*}, \rho(\lambda)=\lambda$ and since $\lim _{\lambda \nearrow \lambda^{*}} \rho(\lambda)=\lambda^{*}$, because $\lambda^{*}=m(0)$ and

$$
\lim _{\alpha \nearrow 0} \sum_{j} \rho_{j}^{*} e^{\alpha \beta j}=\sum_{j} \rho_{j}^{*}=m(0), \quad d \geq 3
$$

we conclude that $\rho(\lambda)$ is continuous and strictly increasing.

Obviously, $\lim _{\lambda \rightarrow \infty} \rho(\lambda)=\lim _{\lambda \rightarrow \infty} \lambda=\infty$. It remains to prove that $\lim _{\lambda \rightarrow-\infty} \rho(\lambda)=0$. By monotonicity, the limit exists. Since $\rho(\cdot)>0, \lambda-\lambda_{0}(\lambda)>0$, so that $\lim _{\lambda \rightarrow-\infty} \lambda_{0}(\lambda)=-\infty$. Then

$$
\lim _{\lambda \rightarrow-\infty} \sum_{j} \rho_{j}^{*} e^{\lambda_{0}(\lambda) \beta j}=\lim _{\alpha \rightarrow-\infty} \sum_{j} \rho_{j}^{*} e^{\alpha \beta j}=0
$$

Theorem G.1 is proved.

Remark. The construction of the minimizing sequence $\underline{\rho}^{(n)}$ in the case $\lambda>\lambda^{*}$ shows that the excess mass $\lambda-\lambda^{*}$ concentrates in the limit on infinitely long loops. 
Let

$$
a(u):=\inf _{\sum_{j} \rho_{j}=u}\left(F_{\lambda}(\underline{\rho})+\lambda u\right)
$$

observing that the r.h.s. does not depend on $\lambda$. Obviously

$$
a(u) \geq \sup _{\lambda}\left\{\lambda u+\inf _{\underline{\rho}} F_{\lambda}(\underline{\rho})\right\}
$$

We will prove that equality actually holds, namely

$$
a(u)=\sup _{\lambda}\left\{\lambda u+\inf _{\underline{\rho}} F_{\lambda}(\underline{\rho})\right\}
$$

which then shows that

$$
a(u)=\sup _{\lambda}\{\lambda u-\pi(\lambda)\}
$$

namely that $a(u)$ is the Legendre transform of $\pi(\lambda)$. In [10] an equivalence of ensembles theorem is proved, namely that

$$
-\lim _{\substack{L \rightarrow \infty \\ N_{L}=\left[L^{d} u\right]}} \frac{1}{L^{d}} \ln Z_{N_{L}, L}^{m f}=\sup _{\lambda}\{\lambda u-\pi(\lambda)\}
$$

which, together with (G.22) shows that

$$
\inf _{\sum_{j} \rho_{j}=u}\left(F_{\lambda}(\underline{\rho})+\lambda u\right)=-\lim _{\substack{L \rightarrow \infty \\ N_{L}=\left[L^{d} u\right]}} \frac{1}{L^{d}} \ln Z_{N_{L}, L}^{m f}
$$

as claimed in the text.

Proof of (G.21) - By (G.3) the inf of $F_{\lambda}(\underline{\rho})$ is achieved on the set $\left\{\underline{\rho}: \sum_{j} \rho_{j}=\lambda-\lambda_{0}\right\}$. Call $\alpha$ the value of $\lambda$ such that

$$
\rho(\alpha)=\alpha-\lambda_{0}(\alpha)=u
$$


(existence and uniqueness follow from Theorem G.1). Then

$$
\begin{aligned}
\sup _{\lambda}\{\lambda u & \left.+\inf _{\underline{\rho}} F_{\lambda}(\underline{\rho})\right\} \geq\left\{\alpha u+\inf _{\underline{\rho}} F_{\alpha}(\underline{\rho})\right\} \\
& =\left\{\alpha u+\inf _{\underline{\rho}: \sum_{j} \rho_{j}=\rho(\alpha)=u} F_{\alpha}(\underline{\rho})\right\}=a(u)
\end{aligned}
$$

which, together with (G.20), proves (G.21).

By general thermodynamic relations, and making explicit now the dependence on $\beta$, the entropy $\sigma(\beta, u)$ and the internal energy, $e(\beta, u)$, are

$$
\sigma(\beta, u)=\beta^{2} \frac{\partial a(\beta, u)}{\partial \beta}, \quad a(\beta, u)=e(\beta, u)-\frac{\sigma(\beta, u)}{\beta}
$$

In our model $e(\beta, u)=\frac{u^{2}}{2}+k(\beta, u)$, with $k(\beta, u)$ the kinetic part of the internal energy. By direct inspection, $\sigma(\beta, u)$ and $k(\beta, u)$ as functions of the particles density $u$, become constant for $u \geq u^{*}$. This is the Bose condensation phenomenon, which is interpreted by saying that the fluid added when increasing the density past $u^{*}$ does not carry entropy nor momentum.

\section{Appendix $\mathbf{H}$}

Let $\underline{\lambda}:=\left\{\lambda_{j}, j>0\right\}$ be the sequence defined by

$$
\lambda_{j}:= \begin{cases}\lambda+\lambda^{\prime} & j \in V_{k}, \\ \lambda & \text { otherwise. }\end{cases}
$$


and

$$
\begin{aligned}
F_{\underline{\lambda}}(\underline{\rho}) & :=\frac{1}{2}\left(\sum_{j>0} \rho_{j}\right)^{2}-\left(\sum_{j>0} \lambda_{j} \rho_{j}\right)-\frac{1}{\beta} S(\underline{\rho}) \\
\pi_{\underline{\lambda}} & :=-\inf _{\underline{\rho}} F_{\underline{\lambda}}(\underline{\rho}) \quad, \quad \rho^{(k)}(\lambda):=\sum_{j \in V_{K}} \rho_{j}(\lambda)
\end{aligned}
$$

Then

$$
\begin{aligned}
& \inf _{\underline{\rho}: \sum_{j \in V_{k}} \rho_{j}=\rho^{(k)}(\lambda)+v} F_{\lambda}(\underline{\rho})= \\
& =\inf _{\underline{\rho}: \sum_{j \in V_{k}} \rho_{j}=\rho^{(k)}(\lambda)+v}\left[F_{\lambda}(\underline{\rho})-\lambda^{\prime}\left(\sum_{j \in V_{k}} \rho_{j}-\left(\rho^{(k)}(\lambda)+v\right)\right)\right] \\
& =\inf _{\underline{\rho}: \sum_{j \in V_{k}} \rho_{j}=\rho^{(k)}(\lambda)+v} F_{\underline{\lambda}}(\underline{\rho})+\lambda^{\prime}\left(\rho^{(k)}(\lambda)+v\right) \\
& \geq-\pi_{\underline{\lambda}}+\lambda^{\prime}\left(\rho^{(k)}(\lambda)+v\right)
\end{aligned}
$$

The equality in line (H.4) holds if $\lambda^{\prime}$ is chosen in such a way that the minimizer sequence $\underline{\rho}_{n}\left(\lambda, \lambda^{\prime}\right)$ satisfies the constraint $\sum_{j \in V_{k}} \rho_{j}=\rho^{(k)}(\lambda)+v$.

The case $V_{k}=N^{+}$follows directly from the arguments used in the appendix $\mathrm{G}$, by changing the chemical potential from $\lambda \rightarrow \lambda+\lambda^{\prime}$, where $\lambda^{\prime}$ is:

$$
\lambda^{\prime}=\rho(\lambda)+v-\lambda+\tilde{\lambda}_{0}
$$

with $\tilde{\lambda}_{0} \equiv \tilde{\lambda}_{0}(\lambda, v)=0$ when $\rho(\lambda)+v \geq \lambda^{*}$, while otherwise is the unique solution of the equation:

$$
\sum_{j} e^{\rho_{j}^{*} \tilde{\lambda}_{0} \beta j}=\rho(\lambda)+v
$$

The minimizer sequence $\left\{\rho_{j}^{(n)}\right\}_{j \in \mathbb{N}^{+}}$is then given by:

$$
\rho_{j}^{(n)}=\rho_{j}^{*} e^{\beta j \tilde{\lambda}_{0}}+\mathbf{1}_{\{j=n\}}\left[\lambda+\lambda^{\prime}-\lambda^{*}\right]_{+}
$$


where we have indicated by $[\cdot]_{+}$the positive part. In particular, if $\lambda>\lambda^{*}$, $v>-\left(\lambda-\lambda^{*}\right)$, then $\lambda^{\prime}=v$.

For $k=2,3,4$, we set $\tilde{\lambda}_{0}(\lambda, v)$ as the solution of the equation

$$
\sum_{j \notin V_{k}} \rho_{j}^{*} e^{\tilde{\lambda}_{0} \beta j}=\lambda-\tilde{\lambda}_{0}-\rho^{(k)}(\lambda)-v
$$

when this solution exists, otherwise we take $\tilde{\lambda}_{0}=0$. Then $\lambda^{\prime}$ is given by the unique solution of the equation:

$$
\sum_{j \in V_{k}} \rho_{j}^{*} e^{\left(\tilde{\lambda}_{0}+\lambda^{\prime}\right) \beta j}=\rho^{(k)}(\lambda)+v
$$

whose existence and uniqueness follows (for any given value of $\tilde{\lambda}_{0}$ ) by monotonicity arguments, since $V_{k}$ is bounded for $k>1$.

Then the minimizer sequence $\left\{\rho_{j}^{(n)}\right\}_{j \in \mathbb{N}^{+}}, n>\max V_{k}$, is given by:

$$
\rho_{j}^{(n)}=\rho_{j}^{*} e^{\beta j\left(\tilde{\lambda}_{0}+\lambda^{\prime}\right)} \mathbf{1}_{j \in V_{k}}+\left(\rho_{j}^{*} e^{\beta j \tilde{\lambda}_{0}}+\mathbf{1}_{\{j=n\}}\left[\lambda-\lambda_{\notin}^{*}-\rho^{(k)}(\lambda)-v\right]_{+}\right) \mathbf{1}_{j \notin V_{k}}
$$

where $\lambda_{\notin}^{*}:=\sum_{j \notin V_{k}} \rho_{j}^{*}$. Notice that, when the positive part is null, $\left\{\rho_{j}^{(n)}\right\}$ does not depends on $n$ and it is actually a minimum.

In the case $k \neq 1, \lambda>\lambda^{*}\left(\Rightarrow \rho^{k}(\lambda)=\lambda_{\epsilon}^{*}\right)$ and $v<\lambda-\lambda^{*}$ :

$$
\pi_{\underline{\lambda}}=\frac{\lambda^{2}}{2}+\frac{1}{\beta} \sum_{j \in V_{K}} \frac{\rho_{j}^{*}}{j} e^{\lambda^{\prime} \beta j}+\frac{1}{\beta} \sum_{j \notin V_{K}} \frac{\rho_{j}^{*}}{j}
$$

where $\lambda^{\prime}$ is the unique solution of $\sum_{j \in V_{k}} \rho_{j}^{*}\left(e^{\lambda^{\prime} \beta j}-1\right)=v$

Collecting equations (G.1) and (H.4), we get:

$$
\inf _{\underline{\rho}: \sum_{j \in V_{k}} \rho_{j}=\rho^{(k)}(\lambda)+v} F_{\lambda}(\underline{\rho})-\inf _{\underline{\rho}} F_{\lambda}(\underline{\rho})=-\pi_{\underline{\lambda}}+\lambda^{\prime}\left(\rho^{(k)}(\lambda)+v\right)+\pi(\lambda)
$$


To prove Theorem 5.2, one has to show (see proof of Theorem E.3 for notation) that

$$
\lim _{L \rightarrow \infty} \frac{\log S_{A, L}(v, \delta)}{\left[\Pi_{A, L}(t)-t \Pi_{A, L}^{\prime}(t)\right]}=\lim _{L \rightarrow \infty}\left[\frac{\frac{1}{\beta L^{d}} \ln P_{\lambda, L}^{m f}\left(\mathcal{A}_{L, \delta}^{(k)}\right)}{-\inf _{\underline{\rho} \in A_{L}^{(k)}} F_{\lambda}(\underline{\rho})+\inf _{\underline{\rho}} F_{\lambda}(\underline{\rho})}\right]
$$

For example, if $\lambda>\lambda^{*}, v<\lambda-\lambda^{*}$ and $k \neq 1$, one has

$$
\begin{aligned}
\inf _{\underline{\rho}: \sum_{j \in V_{k}} \rho_{j}=\rho^{(k)}(\lambda)+v} & F_{\lambda}(\underline{\rho})-\inf _{\underline{\rho}} F_{\lambda}(\underline{\rho})= \\
= & -\frac{1}{\beta} \sum_{j \in V_{k}} \frac{\rho_{j}^{*}}{j}\left(e^{\lambda^{\prime} \beta j}-1\right)+\lambda^{\prime}\left(\rho^{(k)}(\lambda)+v\right)
\end{aligned}
$$

Hence, by using (E.3), (E.4), (E.8) and (E.9), it is easy to check that the two limits are equal in the case $k=4$. The other cases can be treated in a similar way.

\section{Appendix I}

If $k=1$, by the analysis in Appendix $\mathrm{H}$, substituting the value of $\lambda^{\prime}$ as a function of $\lambda$ and $\tilde{\lambda}_{0}$ in (H.4), we get:

$$
\inf _{\underline{\rho}: \sum_{j} \rho_{j}=\rho(\lambda)+v} F_{\lambda}(\underline{\rho})=\frac{1}{2}(\rho+v)^{2}-\left(\lambda-\tilde{\lambda}_{0}\right)(\rho(\lambda)+v)-\frac{1}{\beta} \sum_{j} \frac{\rho_{j}^{*}}{j} e^{\beta j \tilde{\lambda}_{0}}
$$

If $\lambda \neq \lambda^{*}$, this expression is twice differentiable and we get:

$$
\begin{aligned}
\left.\frac{d^{2} \Im(v)}{d v^{2}}\right|_{v=0}=1+\mathbf{1}_{\left\{\lambda<\lambda^{*}\right\}} & {\left[2 \frac{d \tilde{\lambda}_{0}}{d v}+\frac{d^{2} \tilde{\lambda}_{0}}{d v^{2}}(\rho(\lambda)+v)-\frac{d^{2} \tilde{\lambda}_{0}}{d v^{2}} \sum_{j} \rho_{j}^{*} e^{\tilde{\lambda}_{0} \beta j}\right.} \\
& \left.-\left(\frac{d \tilde{\lambda}_{0}}{d v}\right)^{2} \sum_{j} \rho_{j}^{*} \beta j e^{\tilde{\lambda}_{0} \beta j}\right]_{v=0}
\end{aligned}
$$


On the other hand, if $\lambda<\lambda^{*}$ and $v$ is small enough, $\tilde{\lambda}_{0}$ satisfies the equation $\rho(\lambda)+v=\sum_{j} \rho_{j}^{*} e^{\tilde{\lambda}_{0} \beta j}$, so that:

$$
\frac{d \tilde{\lambda}_{0}}{d v} \sum_{j} \rho_{j}^{*} \beta j e^{\tilde{\lambda}_{0} \beta j}=1
$$

and we get

$$
\begin{aligned}
\left.\frac{d^{2} \Im(v)}{d v^{2}}\right|_{v=0} & =1+\mathbf{1}_{\left\{\lambda<\lambda^{*}\right\}}\left[2 \frac{d \tilde{\lambda}_{0}}{d v}+\left(\frac{d \tilde{\lambda}_{0}}{d v}\right)^{2} \sum_{j} \rho_{j}^{*} \beta j e^{\tilde{\lambda}_{0} \beta j}\right]_{v=0} \\
& =1+\mathbf{1}_{\left\{\lambda<\lambda^{*}\right\}} \frac{1}{\sum_{j} \rho_{j}^{*} \beta j e^{\lambda_{0} \beta j}}
\end{aligned}
$$

where we used also the fact that, if $v=0, \tilde{\lambda}_{0}=\lambda_{0}, \lambda_{0}$ being defined as in (G.5).

If $\lambda=\lambda^{*}$, the right and left limit of the derivative of $\tilde{\lambda}_{0}$ in $v=0$ do exist and are given by:

$$
\left\{\begin{array}{l}
\lim _{v \rightarrow 0^{+}} \frac{d \tilde{\lambda}_{0}\left(\lambda^{*}, v\right)}{d v}=0 \\
\lim _{v \rightarrow 0^{-}} \frac{d \tilde{\lambda}_{0}\left(\lambda^{*}, v\right)}{d v}=\lim _{v \rightarrow 0^{-}}\left[\frac{1}{\sum_{j} \rho_{j}^{*} \beta j e^{\tilde{\lambda}_{0}\left(\lambda^{*}, v\right) \beta j}}\right]=\frac{1}{\sum_{j} \rho_{j}^{*} \beta j}
\end{array}\right.
$$

so that, if $d \leq 4$, the second derivative of $\Im(v)$ in $v=0$ does exist and is given by

$$
\left.\frac{d^{2} \Im(v)}{d v^{2}}\right|_{v=0}=\left[1+\frac{\mathbf{1}_{\left\{\lambda<\lambda^{*}\right\}}}{\beta \sum_{j} \rho_{j}^{*} j e^{\lambda_{0}(\lambda) \beta j}}\right]^{-1}
$$

It follows that, if $d=3,4, d^{2} \Im(v) / d v^{2}$ is given by equation (I.6) for any $\lambda$, while, if $d>4$, the same result is true, but only for $\lambda \neq \lambda^{*}$. 


\section{References}

[1] C. Block: in Studies in Statistical Mechanics, J. De Boer and G.E. Uhlenbeck eds. VOl. III, NorthHolland, Amsterdam (1965)

[2] E. Buffet, J.V. Pulè: Fluctuation properties of the imperfect Bose gas, J. Math. Phys. 24, 1608-1616 (1983).

[3] W. Feller: An introduction to Probability Theory and its applications, Vol. II, John Wiley, New York (1971)

[4] R. Feynman: Statistical Mechanics, Benjamin, Reading, MA (1972)

[5] J. Ginibre: Some applications of functional integration in statistical mechanics, in Statistical Mechanics and Field Theory, C. De Witt and R. Stora eds. (Gordon and Breach, New York, 19 70).

[6] K. Huang: Statistical Mechanics, J. Wiles, (1963)

[7] M. Kac, J.M. Luttinger: Bose-Einstein condensationin the presence of impurities, J. Math. Phys. 14, 1626-1628 (1973)

[8] M. Kac, J.M. Luttinger: Bose-Einstein condensationin the presence of impurities II, J. Math. Phys. 15, 183-186 (1974)

[9] J. Kahn: private communication

[10] J.T. Lewis: Why do Bosons condense ? in Statistical Mechanics and Field Theory: Mathematical aspects - Lectures notes in Physics, 257 - 234-256 Springer (1986)

[11] O.Penrose, L.Onsager:Bose condensation and liquid Helium, Phys. Rev.104, 576-583 (1956)

[12] D. Ruelle: Statistical Mechanics, Benjamin, New York (1969)

[13] A.Suto: Percolation transition in the Bose gas: II J. of Physics A: Math.Gen., 35, 6995-7002 (2002)

[14] A.M. Vershik: Statistical mechanics of combinatorial partitions and their limit shapes in Funktsional. Anal. i Prilozhen. 30 19-39, (1996), (Russian); translation in Funct. Anal. Appl. 30, 90-105 (1996).

[15] R.M. Ziff, G.E. Uhlembeck, M. Kac: The ideal Bose-Einstein gas revisited, Reports in Physics 32 169-248 (1967)

G. Benfatto, Dipartimento di Matematica, Università di Roma Tor Vergata, 00133 Roma, Italy

E-mail address: benfatto@mat.uniroma2.it

Marzio Cassandro, Dipartimento di Fisica, Università di Roma la Sapienza, and INFM, Sezione di Roma, 00185 Roma, Italy

E-mail address: Marzio.Cassandro@roma1.infn.it

I. Merola, Dipartimento di Matematica, Università di Roma Tor Vergata, 00133 Roma, Italy

E-mail address: merola@mat.uniroma2.it

E. Presutti, Dipartimento di Matematica, Università di Roma Tor Vergata, 00133 Roma, ItALY

E-mail address: presutti@mat.uniroma2.it 